\title{
Direct numerical simulation of turbulent flow past a trailing edge and the associated noise generation
}

\author{
RICHARD D. SANDBERG AND NEIL D. SANDHAM \\ School of Engineering Sciences, University of Southampton, Southampton SO17 1BJ, UK
}

(Received 16 April 2007 and in revised form 24 September 2007)

Direct numerical simulations (DNS) are conducted of turbulent flow passing an infinitely thin trailing edge. The objective is to investigate the turbulent flow field in the vicinity of the trailing edge and the associated broadband noise generation. To generate a turbulent boundary layer a short distance from the inflow boundary, highamplitude lifted streaks and disturbances that can be associated with coherent outerlayer vortices are introduced at the inflow boundary. A rapid increase in skin friction and a decrease in boundary layer thickness and pressure fluctuations is observed at the trailing edge. It is demonstrated that the behaviour of the hydrodynamic field in the vicinity of the trailing edge can be predicted with reasonable accuracy using triple-deck theory if the eddy viscosity is accounted for. Point spectra of surface pressure difference are shown to vary considerably towards the trailing edge, with a significant reduction of amplitude occurring in the low-frequency range. The acoustic pressure obtained from the DNS is compared with predictions from two- and three-dimensional acoustic analogies and the classical trailing-edge theory of Amiet. For low frequencies, two-dimensional theory succeeds in predicting the acoustic pressure in the far field with reasonable accuracy due to a significant spanwise coherence of the surface pressure difference and predominantly two-dimensional sound radiation. For higher frequencies, however, the full three-dimensional theory is required for an accurate prediction of the acoustic far field. DNS data are used to test some of the key assumptions invoked by Amiet for the derivation of the classical trailing-edge theory. Even though most of the approximations are shown to be reasonable, they collectively lead to a deviation from the DNS results, in particular for higher frequencies. Moreover, because the three-dimensional acoustic analogy does not provide significantly improved results, it is suggested that some of the discrepancies can be attributed to the approach of evaluating the far-field sound using a Kirchhoff-type integration of the surface pressure difference.

\section{Introduction}

For modern aircraft in approach, fan noise and airframe noise are important contributors to the perceived sound on the ground. Ffowcs Williams \& Hall (1970) demonstrated that turbulent fluctuations subject to a scattering process at a sharp edge of a solid body radiate three orders of magnitude stronger $\left(u^{5}\right.$-scaling) than turbulent fluctuations in free space $\left(u^{8}\right.$-scaling) (Lighthill 1952a, b). For this reason, trailing edges are one of the main sources of airframe noise and fan noise, in particular for lower Mach numbers. Therefore, a detailed understanding of the underlying physical 
mechanisms in the generation of trailing-edge noise would be beneficial for the design of quieter aircraft and propulsion systems.

Because of the importance of trailing-edge noise, several theories have been developed, relying on various degrees on empiricism. An exhaustive review of trailingedge theories was conducted by Howe (1978). He identified three categories, namely theories based on Lighthill's acoustic analogy, theories based on the solution of special problems approximated by linearized equations, and ad hoc models. Howe concluded that all these methods, when interpreted properly, essentially gave the same results at vanishingly small Mach numbers. The theoretical results were supported by trailing-edge noise experiments on a NACA-0012 airfoil (Brooks \& Hodgson 1981). From the second category, the theory of Amiet (1976b) appears to be an attractive approach as the far-field noise can be predicted with the convecting surface pressure spectrum upstream of the trailing edge being the required input rather than having to provide the turbulence quadrupole sources in full.

With the recent dramatic growth in computing power, conducting numerical simulations in order to compute aerodynamically generated sound has become increasingly feasible. Nevertheless, directly computing aerodynamic sound remains challenging for several reasons. First, the extent of the acoustic field is considerably larger than the relevant flow field. The need for a large computational domain in combination with the requirement of resolving small scales in the hydrodynamic field and all relevant acoustic waves in the radiation field demands significant computational resources. Secondly, the energy of the acoustic field is considerably smaller than that of the hydrodynamic field, requiring numerical schemes with minimal amounts of artificial dissipation. Finally, special care needs to be taken for freespace boundary conditions so that no spurious pressure reflections contaminate the region of interest (Colonius, Lele \& Moin 1993). Because of these difficulties, many computations of trailing-edge noise have used hybrid approaches, where a hydrodynamic simulation of the relevant flow field is coupled with an acoustic solver for the far field. Typically, to further reduce computational cost, large-eddy simulations (LES) are conducted of the flow field (see Wang \& Moin 2000; Oberai, Roknaldin \& Hughes 2002; Marsden, Bogey \& Bailly 2006a).

Nevertheless, solving the full unsteady Navier-Stokes equations to directly compute both the far field sound and the near-field hydrodynamics would be desirable. Using direct numerical simulations (DNS), difficulties with hybrid approaches, such as the coupling of different numerical methods, storage of intermediate data (e.g. Manoha et al. 2002), and the sensitivity to the position of integration surfaces (see Singer et al. 2000) can be avoided. Also, uncertainties in subgrid-stress models for the small-scale structures in LES (see Seror et al. 2001; Bodony \& Lele 2002) and problems with predicting the laminar-turbulent transition (Marsden, Bogey \& Bailly 2006b) are eliminated.

Thus, for the present investigation, DNS are employed to investigate the hydrodynamic field in the vicinity of the trailing edge and the associated broadband trailing-edge noise. To reduce the computational cost of the simulations, a thin flat plate is considered as a generic example of a sharp trailing edge (TE). A turbulent inflow generation technique that exploits the dynamical features of the outer and inner part of the boundary layer is employed on the top side of the flat plate to produce a turbulent boundary layer that convects over the TE. On the bottom side of the plate, a laminar compressible boundary layer is prescribed. The set-up of the simulation exhibits two key advantages for the acoustic investigation. First, the classical theory of Amiet (1976b) considers compressible turbulent flow over a flat 
plate, making direct comparison of the DNS data with the inviscid theory viable. Moreover, by introducing a turbulent boundary layer on one side of the plate only, the trailing-edge noise can easily be separated from other sources of sound. On the bottom side of the plate, only the scattered pressure field can be observed while on the top side the scattered pressure field is superposed with the incident pressure field.

In addition to investigating the noise generation mechanisms and performing a comparison with the classical theory of Amiet, the DNS data can also be used to analyse the behaviour of the turbulent boundary layer in the vicinity of the trailing edge. It is well known that classical boundary-layer theory breaks down when streamwise gradients occur on a length scale similar to the boundary layer thickness. The sudden change from a no-slip boundary condition for the velocity components on the surface to non-zero values in the developing wake induces a streamwise pressure gradient in the vicinity of the trailing edge. Stewartson (1968) showed that the boundary layer approximation fails within a distance $O\left(l R e_{l}^{3 / 4}\right)$ of the trailing edge, where $l$ denotes the length of the plate and the Reynolds number is based on the free-stream velocity and the plate length. Stewartson (1968) and Messiter (1970) demonstrated that the appropriate equations for the vicinity of the trailing edge are the full Navier-Stokes equations. They derived asymptotic expansions of the flow variables in the neighbourhood of the trailing edge and found a disturbance structure known as the triple-deck structure (for a comprehensive review of triple-deck theory, see e.g. Smith 1982). Subsequently, the triple-deck approach has frequently been employed to investigate the flow behaviour close to trailing edges (see Daniels 1977; Gajjar \& Türkyilmazog̃lu 2000), although the analyses have almost exclusively been restricted to laminar flows. In addition, the asymptotic approach inherently assumes that the Reynolds number tends to infinity. However, even though in most applications the flow is turbulent by the time the trailing edge is reached, the Reynolds numbers frequently are moderate. Thus, the behaviour of a turbulent flow in the vicinity of a trailing edge at moderate Reynolds number remains unclear and will be investigated in the present paper.

The paper is structured as follows. In $\S 2$ the governing equations for the direct numerical simulations and the prediction of the acoustic pressure both in two and three dimensions are presented. Amiet's classical trailing edge theory (Amiet 1976b) and the key assumptions made in its derivation are highlighted. Section 3 describes the numerical tools employed to solve the governing equations. The technique employed for the generation of a turbulent boundary layer is introduced in $\S 4$. In $\S 5$, first the hydrodynamic field is scrutinized, focusing on the behaviour of the turbulent boundary layer as it approaches the trailing edge, and a comparison is made with the asymptotic theories of Stewartson (1968) and Messiter (1970). Then, the acoustic pressure predicted by two- and three-dimensional acoustic analogies and Amiet's classical trailing-edge theory is compared with DNS data in the frequency domain. The paper concludes with a discussion of the most important results in $\S 6$.

\section{Governing equations}

\subsection{Direct numerical simulations}

The flow under consideration is governed by the full compressible Navier-Stokes equations. The fluid is assumed to be an ideal gas with constant specific heat coefficients. For simplicity, all equations in this section are presented in tensor notation. All dimensional quantities, denoted by an asterisk, are made dimensionless using the flow quantities at a reference location in the flow; here the free-stream/inflow 
location is used. The semi-chord $b^{*}$ was chosen as the reference length which here is half the distance from the end of the inflow sponge to the trailing edge. The non-dimensionalization results in the Reynolds number $R e=\rho_{\infty}^{*} u_{\infty}^{*} b^{*} / \mu_{\infty}^{*}$, the Mach number $M=u_{\infty}^{*} / c_{\infty}^{*}$, and the Prandtl number $\operatorname{Pr}=\mu_{\infty}^{*} c_{p}^{*} / \kappa_{\infty}^{*}$. The non-dimensional continuity, momentum and energy equations are

$$
\begin{gathered}
\frac{\partial \rho}{\partial t}+\frac{\partial}{\partial x_{k}}\left(\rho u_{k}\right)=0, \\
\frac{\partial}{\partial t}\left(\rho u_{i}\right)+\frac{\partial}{\partial x_{k}}\left[\rho u_{i} u_{k}+p \delta_{i k}-\tau_{i k}\right]=0, \\
\frac{\partial}{\partial t}(\rho E)+\frac{\partial}{\partial x_{k}}\left[\rho u_{k}\left(E+\frac{p}{\rho}\right)+q_{k}-u_{i} \tau_{i k}\right]=0,
\end{gathered}
$$

where the total energy is defined as $E=T /\left[\gamma(\gamma-1) M^{2}\right]+\frac{1}{2} u_{i} u_{i}$ with $\gamma=1.4$. The molecular stress-tensor and the heat-flux vector are computed as

$$
\tau_{i k}=\frac{\mu}{R e}\left(\frac{\partial u_{i}}{\partial x_{k}}+\frac{\partial u_{k}}{\partial x_{i}}-\frac{2}{3} \frac{\partial u_{j}}{\partial x_{j}} \delta_{i k}\right), \quad q_{k}=\frac{-\mu}{(\gamma-1) M^{2} \operatorname{PrRe}} \frac{\partial T}{\partial x_{k}},
$$

respectively, where the Prandtl number is assumed to be constant at $\operatorname{Pr}=0.72$. The molecular viscosity $\mu$ is computed using Sutherland's law (see White 1991), setting the ratio of the Sutherland constant over free-stream temperature to 0.36867 . To close the system of equations, the pressure is obtained from the non-dimensional equation of state $p=(\rho T) /\left(\gamma M^{2}\right)$.

\subsection{Acoustic pressure prediction}

The starting point is Goldstein's formulation of the acoustic analogy (Goldstein 1976), which represents the fundamental equation governing the generation of aerodynamic sound in the presence of solid boundaries in a moving reference frame. The far field and the surface coordinates $x_{i}$ and $y_{i}$, respectively, are non-dimensionalized with the semi-chord $b^{*}$. The streamwise, wall-normal and spanwise directions are denoted by the subscripts $i=1,2,3$, respectively. A plate with zero thickness is specified to be semi-infinite, i.e. the leading edge is not considered. However, the integration of sources along the plate surface is conducted over a finite length, namely $-2 \leqslant y_{1} \leqslant 0$. It is assumed that the volume-quadrupole sources generated by the shear-stress components in the boundary layer are negligible compared with the dipole sources on the surface and that the surface is rigid. Performing a Fourier transform in time, the acoustic pressure at a single frequency due to the surface pressure difference $\Delta p_{t}$ becomes

$$
p\left(x_{i}, \omega\right)=-\int_{0}^{d} \int_{-2}^{0} \Delta p_{t}\left(y_{i}, \omega\right) \frac{\partial}{\partial y_{2}} G\left(x_{i}, y_{i}, \omega\right) \mathrm{d} y_{1} \mathrm{~d} y_{3},
$$

where $\omega$ is the frequency, $G$ denotes the radiation Green's function, and $d$ is the spanwise width of the surface, non-dimensionalized with the semi-chord.

\subsubsection{Three-dimensional theory}

When dealing with turbulent flows, statistical quantities need to be used, such as the cross-power spectral density (Amiet 1975). Following Amiet (1975), the cross-power 
spectral density (PSD) is defined as

$$
\begin{aligned}
S_{p p}\left(x_{i}, \omega\right)= & \lim _{\tau \rightarrow \infty} \frac{\pi}{\tau} \mathscr{E}\left\{p\left(x_{i}, \omega\right) \bar{p}\left(x_{i}, \omega\right)\right\} \\
= & \lim _{\tau \rightarrow \infty} \frac{\pi}{\tau} \int_{0}^{d} \int_{-2}^{0} \int_{0}^{d} \int_{-2}^{0} \mathscr{E}\left\{\Delta p_{t}\left(y_{i}, \omega\right) \frac{\partial}{\partial y_{2}} G_{3 D}\left(x_{i}, y_{i}, \omega\right) \Delta \bar{p}_{t}\left(z_{i}, \omega\right)\right. \\
& \left.\times \frac{\partial}{\partial z_{2}} \bar{G}_{3 D}\left(x_{i}, z_{i}, \omega\right)\right\} \mathrm{d} y_{1} \mathrm{~d} y_{3} \mathrm{~d} z_{1} \mathrm{~d} z_{3},
\end{aligned}
$$

where $\mathscr{E}\{\}$ is the expected value, or ensemble average (the numerical implementation is discussed in $\S 5.2 .1$ ), an overlined quantity is the complex conjugate, $\tau$ is the sampling interval, and it is assumed that the three-dimensional Green's function $G_{3 D}\left(x_{i}, y_{i}, \omega\right)$ is not time-dependent. The total surface pressure difference is the sum of the incident pressure difference and the scattered pressure difference

$$
\Delta p_{t}\left(y_{i}, \omega\right)=\Delta p_{i}\left(y_{i}, \omega\right)+\Delta p_{s}\left(y_{i}, \omega\right) .
$$

The 'incident' pressure field refers to pressure fluctuations that convect over the plate in the downstream direction and are scattered at the trailing edge. From compressible DNS, the entire time-series of the total pressure difference on the surface is available and equation (2.6) can be integrated directly to obtain the acoustic pressure field. This method is denoted as using the three-dimensional acoustic analogy in the following.

Because of the limit of computational resources, the computational domain for the trailing-edge simulations was truncated at a distance from the trailing edge that does not satisfy the assumption of $x_{i} \gg y_{i}$. Therefore, no far-field assumption is made in the current investigation and the wall-normal derivative of the radiation Green's function, adopted from (Amiet 1975), is retained as

$$
\frac{\partial G_{3 D}\left(x_{i}, y_{i}, \omega\right)}{\partial y_{2}}=-\frac{y_{2}-x_{2}}{4 \pi R_{3 D}^{2}}\left[\frac{\beta^{2}}{R_{3 D}}-\mathrm{i} \omega M\right] \exp \left(\mathrm{i} \mu_{0}\left[M\left(y_{1}-x_{1}\right)+R_{3 D}\right]\right),
$$

where $R_{3 D}=\sqrt{\left(y_{1}-x_{1}\right)^{2}+\beta^{2}\left[\left(y_{2}-x_{2}\right)^{2}+\left(y_{3}-x_{3}\right)^{2}\right]}$, and the reduced frequency is $\mu_{0}=\omega^{*} b^{*} /\left(c_{\infty}^{*} \beta^{2}\right)$ with $\beta=\sqrt{1-M^{2}}$.

\subsubsection{Classical theory of Amiet}

In Amiet's classical theory a number of additional assumptions are made (beyond using a Kirchoff-type integration of the surface pressure difference) to arrive at the final expression for the far-field spectrum for an observer in the $x_{3}=0$ plane.

First, it is assumed that only an incident pressure field is known. The total pressure field at the trailing edge is determined from the incident pressure field through a transfer function (Amiet 1976a). The scattered surface pressure difference is evaluated through

$$
\Delta p_{s}\left(y_{1}, K_{x}\right)=H_{S}\left(y_{1}, K_{x}\right) p_{i}\left(y_{1}, K_{x}\right)
$$

with

$$
H_{S}\left(y_{1}, K_{x}\right)=\left\{(1+\mathrm{i}) C\left[-\left(\mu_{0}(1+M)+K_{x}\right) y_{1}\right]-1\right\},
$$

where $C$ is a combination of Fresnel integrals and the streamwise wavenumber is defined as $K_{x}=\omega^{*} b^{*} / U_{c}^{*}$ with $U_{c}^{*}$ being the convection speed of the disturbance. Adding the scattered pressure field to the incident pressure field, the total pressure difference becomes

$$
\Delta p_{t}\left(y_{1}, K_{x}\right)=H_{D}\left(y_{1}, K_{x}\right) p_{i}\left(y_{1}, K_{x}\right)=\left[1+H_{S}\left(y_{1}, K_{x}\right)\right] p_{i}\left(y_{1}, K_{x}\right) .
$$


The derivation of the surface pressure jump function $H_{S}$ assumes the unsteady Kutta condition to hold. Note that the total surface pressure difference is also a function of the convection speed when computed using the surface pressure jump function.

The validity of the surface pressure jump function $H_{S}$ can be investigated using DNS data. In Sandberg, Sandham \& Joseph (2007) the total surface pressure difference obtained from two-dimensional trailing-edge simulations was compared to $\Delta p_{t}$ computed using (2.10), where the incident pressure was provided from an additional DNS of a flat-plate boundary layer without a trailing edge. Here, owing to the significant cost of performing DNS of turbulent flows, a different approach is chosen. The incident pressure field $p_{i}$ is extracted from the trailing-edge DNS data. Because the pressure signal of the top surface is composed of the incident and the scattered pressure fields while the bottom surface only contains the scattered pressure field, $p_{i}$ can be computed by subtracting the bottom surface pressure from the top surface pressure. Equation (2.10) can then be used to compute the total surface pressure difference $\Delta p_{t}$. It should be noted that for this approach to yield accurate results for the incident pressure the scattered pressure field must not be substantially modified by the turbulent boundary layer on the top side of the plate. Otherwise, the difference between the scattered pressure field affected by the turbulence and the scattered field from the laminar side would be added to the incident pressure field.

Amiet (1976b) goes a step further and assumes that the incident pressure can be represented as $p_{i}\left(y_{1}, K_{x}\right)=p_{0} \mathrm{e}^{\mathrm{i} \omega\left(t-y_{1} / U_{c}\right)}$, thus, the total pressure jump (normalized with $p_{0}$ ) is

$$
g\left(y_{1}, K_{x}\right)=H_{S}\left(y_{1}, K_{x}\right) \mathrm{e}^{-\mathrm{i}\left(\omega y_{1} / U_{c}\right)} .
$$

By invoking the far-field assumption $x_{i} \gg y_{i}$ the wall-normal derivative of the radiation Green's function can be written as

$$
\frac{\partial G_{3 D}\left(x_{i}, y_{i}, \omega\right)}{\partial y_{2}}=\frac{\mathrm{i} \omega M x_{2}}{4 \pi \sigma^{2}} \exp \left(\mathrm{i} \mu_{0}\left[\sigma-\frac{x_{1} y_{1}+\beta^{2} x_{3} y_{3}}{\sigma}+M\left(y_{1}-x_{1}\right)\right]\right),
$$

where $\sigma=\sqrt{x_{1}^{2}+\beta^{2}\left(x_{2}^{2}+x_{3}^{2}\right)}$. The main benefit of this simplification is that $\sigma$ is independent of the surface coordinates $y_{i}$ and can therefore be factored out of the integral when (2.12) is substituted into (2.6).

Amiet (1976b) defines

$$
\mathscr{L}\left(x_{1}, K_{x}\right)=\int_{-2}^{0} g\left(y_{1}, K_{x}\right) \exp \left(-\mathbf{i} \mu_{0} y_{1}\left(M-\frac{x_{1}}{\sigma}\right)\right) \mathrm{d} y_{1}
$$

for which he derives a closed-form solution. However, only the scattered pressure part was accounted for in the original paper and the contribution of the incident pressure field to the total pressure jump is accounted for in the corrected solution for $\mathscr{L}\left(x_{1}, K_{x}\right)$ in Amiet (1978). Finally, assuming a large span $d$ of the plate relative to the relevant spanwise fluctuations and a frozen turbulence spectrum, the far-field spectrum for an observer in the $x_{3}=0$ plane becomes

$$
S_{p p}(\underline{x}, \omega)=\left(\frac{\omega M x_{2}}{2 \pi \sigma^{2}}\right)^{2} d\left|\mathscr{L}\left(x_{1}, K_{x}\right)\right|^{2} l_{x_{3}}(\omega) S_{q q}(\omega, 0) .
$$

The frequency-dependent spanwise correlation length $l_{x_{3}}(\omega)$ is defined as

$$
l_{x_{3}}(\omega)=\frac{1}{S_{q q}(\omega, 0)} \int_{0}^{\infty} S_{q q}\left(\omega, x_{3}\right) \mathrm{d} x_{3}
$$


and is approximated in Amiet $(1976 b)$ by $l_{x_{3}}(\omega)=2.1 U_{c} / \omega$. The non-dimensional frozen power spectrum $S_{q q}(\omega, 0)$ of a turbulent boundary layer is approximated by the empirical expression

$$
S_{q q}(\omega, 0)=\frac{\delta_{1}}{2} 1 \times 10^{-5} /\left(1+\tilde{\omega}+0.217 \tilde{\omega}^{2}+0.00562 \tilde{\omega}^{4}\right), \quad 0.1<\tilde{\omega}<20,
$$

with $\tilde{\omega}=\omega \delta_{1}$. The displacement thickness is approximated by $\delta_{1}=0.094 R e_{c}^{-1 / 5}$ where $R e_{c}$ is the Reynolds number based on chord.

\subsubsection{Two-dimensional single-frequency theory}

In the current investigation, the turbulence may not be entirely random because forcing (with specific frequencies) is employed to generate it. An attempt is therefore made to use deterministic quantities such as point spectra. Another deviation from Amiet's classical theory is the fact that only a narrow spanwise domain was simulated (owing to limitations in computational resources) using periodic boundary conditions. The sound radiation is therefore expected to be predominantly two-dimensional, as in Oberai et al. (2002). Hence, the previously presented modification of Amiet's theory accounting for two-dimensional sound radiation of single-frequency disturbances (Sandberg et al. 2007) is employed.

The main modification to the original theory consisted of replacing the threedimensional Green's function with a two-dimensional Green's function to account for radiation in two dimensions only. Inserting the two-dimensional Green's function (Sandberg et al. 2007) in (2.5) yields the acoustic pressure for a single frequency

$$
p\left(x_{i}, \omega\right)=\frac{\mathrm{i} x_{2} \omega M}{4 \beta} \int_{-2}^{0} \Delta p_{t}\left(y_{1}, \omega\right) \frac{1}{R_{2 D}} H_{1}^{(2)}\left\{\mu_{0}\left[M\left(y_{1}-x_{1}\right)+R_{2 D}\right]\right\} \mathrm{d} y_{1},
$$

where $H_{1}^{(2)}$ denotes a first-order Hankel function of the second kind. Note that, in contrast to the original theory, no far-field approximation has been made for the two-dimensional theory. In the two-dimensional case, the total surface pressure difference is a function of frequency and the streamwise coordinate only. However, the three-dimensional DNS provides pressure data over the entire surface. Here, the spanwise average (the zeroth spanwise mode) is chosen to evaluate the surface pressure difference. Using the time series of the surface pressure difference from DNS and directly integrating equation (2.17) is denoted as using the two-dimensional acoustic analogy in the following.

\section{Numerical method}

Several numerical codes are used: one solves the full compressible Navier-Stokes equations while the others solve for the acoustic pressure as a function of the surface pressure difference in either two or three dimensions.

\subsection{Navier-Stokes code}

The compressible Navier-Stokes equations are discretized using a high-orderaccurate numerical scheme applicable to general geometries. The extension to general coordinates is achieved through metric terms pre-multiplying the derivatives of the governing equations. No upwinding, artificial dissipation or explicit filtering are employed. Stability is, rather, achieved through appropriate conditioning of the governing equations, such as an entropy-splitting approach that splits the nonlinear terms into conservative and non-conservative parts and a Laplacian formulation of the 
viscous and heat conduction terms. The latter is used to avoid odd-even decoupling when using central finite-difference schemes. In addition, compatible spatial difference operators for the interior and boundary points are employed. The Navier-Stokes code has been thoroughly tested (see e.g. Sandberg et al. 2007). Further details on the fundamental numerical approach are given in Sandham, Li \& Yee (2002).

\subsubsection{Numerical treatment of the trailing edge}

The present paper is concerned with the turbulent flow field in the vicinity of a sharp trailing edge and the associated broadband noise generation. Hence the numerical treatment of the geometric singularity is of particular importance. The infinitely thin flat plate is realized by specifying no-slip boundary conditions on a single grid line, extending from the inflow boundary to $y_{1}=0$. Because the thermodynamic variables $p, \rho$ and $T$ are permitted to have different values on the top and bottom surfaces, two values of each quantity are stored for each grid point representing the plate, including the point at $y_{1}=0$. For convenience, we refer to the point $y_{1}=0$ as the trailing edge. In practice, the change from the no-slip boundary condition to the first free-space point will occur at a small $\Delta y_{1}$ downstream of $y_{1}=0$. For the wall-normal derivatives, a boundary finite-difference scheme according to Carpenter, Nordström \& Gottlieb (1999) is employed for the wall and wall-adjacent points along the plate. Starting with the first grid point downstream of the trailing edge, central differences are utilized to evaluate wall-normal derivatives. For streamwise derivatives on $x_{2}=0$ three different approaches are necessary. From the inflow boundary to the trailing edge, derivatives are computed for both the top and the bottom surface individually using five-point central difference stencils, and stored separately. For the first two grid points downstream of the trailing edge, the streamwise derivatives are determined employing the same central finite-difference scheme, with the function values of the upstream points being specified as the average of the top and bottom surface. All streamwise derivatives downstream of these two special points are computed using the same finite difference scheme as for the entire computational domain. One-sided derivative schemes were also tested for the points up- and downstream of the TE, but the above described approach was found to yield the smoothest results in the vicinity of the trailing edge.

\subsubsection{Free-space boundary conditions}

At the inflow and free-stream boundaries, characteristic boundary conditions are used in combination with traditional sponges. A non-reflecting zonal boundary condition is used to avoid spurious pressure oscillations from the outflow boundary, which is subject to the passage of nonlinear disturbances, i.e. vortical structures. The method is based on commonly used characteristic boundary conditions. Here, incoming characteristics are ramped to zero in a buffer zone as opposed to merely setting them to zero at the boundary (see Sandberg \& Sandham 2006). Crucially, in contrast to most other zonal approaches, the method is free of coefficients that require calibration and only the length of the fringe zone needs to be specified. The zonal characteristic boundary condition has been shown to be highly effective for a variety of two-dimensional flow simulations (cf. Sandberg \& Sandham 2006; Sandberg, Jones \& Sandham 2006), but is applied to a three-dimensional simulation for the first time in the present investigation.

\subsection{Acoustic analogies}

For the prediction of the acoustic pressure, equations (2.6) and (2.17) are solved numerically using a fourth-order-accurate integration. The surface is discretized with 
a non-uniform grid, clustering most points in the vicinity of the trailing edge to reduce computational cost. A thorough validation of the numerical codes was performed in Sandberg et al. (2007). In addition, (2.14) was evaluated using the values provided in Amiet (1976b) and the result shown in figure 1 of Amiet (1978) was reproduced.

\section{Turbulent inflow generation}

The surface of the infinitely thin plate extends upstream to the inflow boundary, i.e. the leading edge is not included in the numerical simulations. Therefore, turbulent inflow conditions need to be prescribed. In order to fully develop the turbulence a short distance from the inflow boundary, the deterministic approach of Sandham, Yao \& Lawal (2003) is employed. An analytic turbulent mean flow profile according to Spalding (1961) is prescribed at the inflow boundary. Inner- and outer-layer disturbances, consisting of waves with specific spanwise wavelengths, frequencies and phase information are then superposed onto the turbulent mean profile for each Runge-Kutta step of the simulation. The inner disturbances represent low-speed, high-amplitude lifted streaks while the outer disturbances can be associated with coherent outer-layer vortices. The wall-normal shape of the disturbances is chosen such that the superposition of all disturbances resembles turbulent intensity profiles from a boundary layer. The disturbances take the form

$$
\begin{aligned}
& u_{1}^{\text {inner }}=c_{1,0} y_{2}^{+} \exp \left(-y_{2}^{+} / y_{2_{p, 0}^{+}}^{+}\right) \sin \left(\omega_{0} t\right) \cos \left(\beta_{0} y_{3}+\phi_{0}\right), \\
& u_{2}^{\text {inner }}=c_{2,0}\left(y_{2}^{+}\right)^{2} \exp \left(-\left(y_{2}^{+} / y_{2_{p, 0}}^{+}\right)^{2}\right) \sin \left(\omega_{0} t\right) \cos \left(\beta_{0} y_{3}+\phi_{0}\right), \\
& u_{1}^{\text {outer }}=\sum_{j=1}^{3} c_{1, j} y_{2} / y_{2_{p, j}} \exp \left(-y_{2} / y_{2_{p, j}}\right) \sin \left(\omega_{j} t\right) \cos \left(\beta_{j} y_{3}+\phi_{j}\right), \\
& u_{2}^{\text {outer }}=\sum_{j=1}^{3} c_{2, j}\left(y_{2} / y_{2_{p, j}}\right)^{2} \exp \left(-\left(y_{2} / y_{2_{p, j}}\right)^{2}\right) \sin \left(\omega_{j} t\right) \cos \left(\beta_{j} y_{3}+\phi_{j}\right),
\end{aligned}
$$

where $j$ denotes a mode, $y_{2}^{+}$is the wall-normal coordinate in wall units, $c_{i, j}$ are constants, $\omega_{j}$ are forcing frequencies, $\beta_{j}$ are spanwise wavenumbers, and $\phi_{j}$ denote phase shifts. The spanwise velocity disturbances are obtained by applying a divergence-free condition. Several precursor boundary layer simulations were conducted employing the turbulent inflow generation technique with varying parameters to establish the best choice of coefficients to obtain the desired $R e_{\delta_{1}^{T E}} \approx 2000$ at the trailing edge. For the DNS of turbulent trailing edge flow, the original parameters (Sandham et al. 2003) were modified to the values given in table 1. Moreover, in the current simulation no random noise was added to break remaining symmetries in order to reduce the noise generated by the turbulent inflow generation technique.

\section{Results}

Direct numerical simulations were conducted of compressible turbulent flow at $M=0.6$ over an infinitely thin plate with a trailing edge. The aim of these simulations is to investigate the hydrodynamic near field in the vicinity of the trailing edge and to examine the trailing edge broadband noise generation. The computational domain has the dimensions $-2.18 \leqslant x_{1} \leqslant 1.45,-2.91 \leqslant x_{2} \leqslant 2.91$, and $0 \leqslant x_{3} \leqslant 0.145$. It is discretized using 2001 and 609 non-equidistantly spaced points in the $x_{1}$ - and 


\begin{tabular}{lcccccccc} 
& $j$ & $c_{1, j}$ & \multicolumn{1}{c}{$c_{2, j}$} & $\omega_{j}$ & $\beta_{j}$ & $\phi_{j}$ & $y_{2_{p, j}}^{+}$ & $y_{2_{p, j}}$ \\
inner & 0 & 0.1 & -0.0016 & 1.0 & $\pi$ & 0.0 & 12 & - \\
outer & 1 & 0.3 & -0.06 & 2.5 & $0.75 \pi$ & 0.0 & - & 0.016 \\
& 2 & 0.3 & -0.06 & 1.25 & $0.5 \pi$ & 0.1 & - & 0.025 \\
& 3 & 0.3 & -0.06 & 0.625 & $0.25 \pi$ & 0.15 & - & 0.044
\end{tabular}

TABLE 1. Parameters for turbulent inflow generation for a trailing-edge flow.

$x_{2}$-directions, respectively, with the finest resolution at the trailing edge. 620 streamwise points are used for the singular plate at $y_{2}=0$ which extends from the inflow boundary to the trailing edge at $y_{1}=0$. Recall that $x_{i}$ denotes the radiation field coordinates while $y_{i}$ is associated with the plate surface coordinate. In the spanwise direction, 87 equidistantly spaced points are used. This results in a total of $106 \times 10^{6}$ grid points.

\subsection{Hydrodynamic field}

The turbulent inflow generation method is applied to the top boundary layer, while the bottom boundary layer is initialized using a compressible laminar similarity solution. This setup was chosen to enable the separation of trailing-edge noise from sound generated by other sources. On the top surface, the trailing-edge noise will inevitably be superposed with noise from the turbulent boundary layer itself and possibly with noise generated by the turbulent inflow generation. On the bottom side, on the other hand, only acoustic pressure fluctuations due to the scattering at the trailing edge will be present. A zonal characteristic boundary condition (Sandberg \& Sandham 2006) is used at the outflow boundary condition (for $x_{1} \geqslant 1.09$ ), and a traditional sponge employing a dissipation term is used for the upper and lower free-stream boundaries $\left(\left|x_{2}\right| \geqslant 2.18\right)$, and the inflow boundary $\left(-2.18 \leqslant x_{1} \leqslant-2\right)$.

The application of the sponge at the inflow is not trivial. On the one hand, reflections from upstream-travelling sound waves and acoustic disturbances produced by the turbulent inflow generation need to be attenuated. On the other hand, the disturbances specified at the inflow must not be fully damped so that a turbulent boundary layer can develop. The approach currently taken is to ramp the sponge in the wall-normal direction, i.e. no sponge is applied between the wall and $\delta_{1}$, and the sponge is then ramped to full strength between $\delta_{1}$ and the boundary layer thickness. In spite of being careful not to fully remove the inflow disturbances, instantaneous iso-surfaces of the second invariant of the velocity gradient tensor on the top surface (figure 1a) illustrate that the inflow sponge still considerably damps the inflow fluctuations. In particular the disturbances introduced in the outer layer are overly attenuated so that the turbulent breakdown of the high-amplitude streaks is delayed, leading to a transition region clearly visible in figure $1(a)$.

This transition region extends over roughly one third of the plate length, which is confirmed by a plot of skin friction, shown in figure $2(b)$. The skin friction decreases drastically within the inflow sponge region and then increases steadily until the flow has fully transitioned to turbulence at $y_{1} \approx-1.4$. Within the inflow sponge, the displacement thickness rises quickly and then approaches the 1/7 power-law prediction (Schlichting 1979) in the transition region. The displacement thickness increases over the plate and the Reynolds number based on displacement thickness reaches $\operatorname{Re}_{\delta_{1}^{T E}}=2222$, slightly higher than originally intended. On the laminar lower surface, $\operatorname{Re}_{\delta_{1}^{T E}}=2133$ at the trailing edge. Figure $1(b)$ shows instantaneous contours of the spanwise vorticity component at $y_{3}=0.727$ in the vicinity of the trailing edge. 


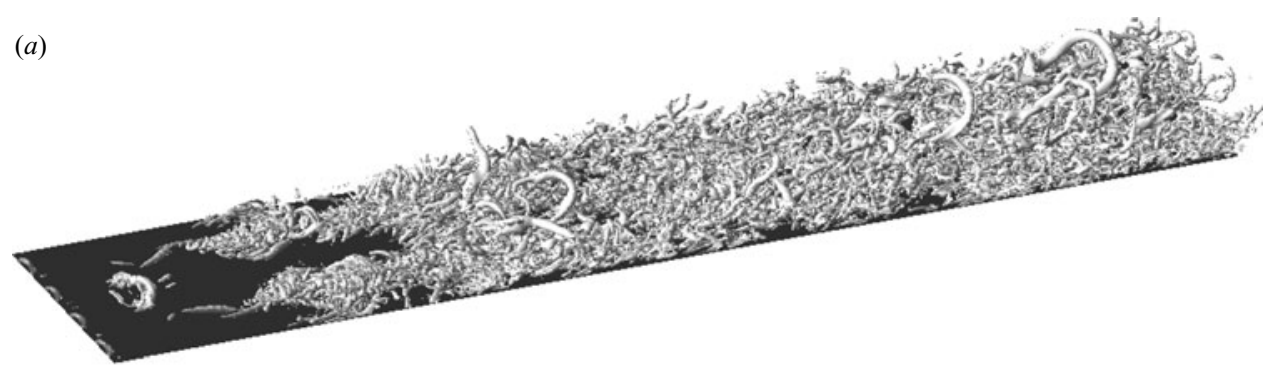

(b)

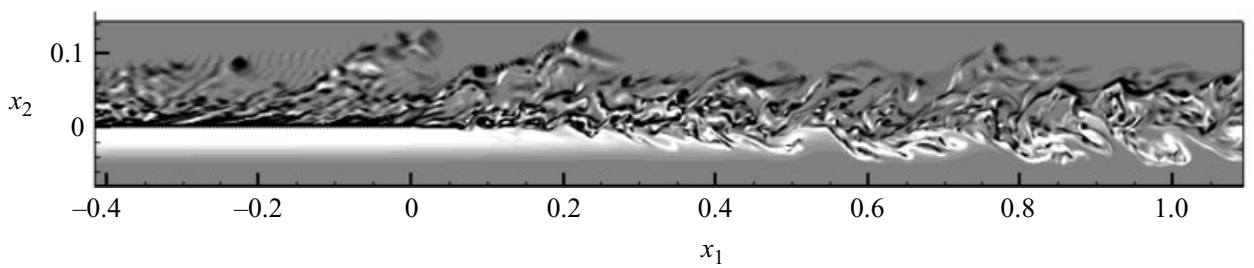

FIGURE 1. (a) Instantaneous iso-surfaces of the second invariant of the velocity gradient tensor on the top surface of the singular plate, and $(b)$ instantaneous contours in the range $[-4 ; 4]$ of the spanwise vorticity component at $x_{3}=0.0725$ in the vicinity of the trailing edge; flow from left to right, $t=96$.
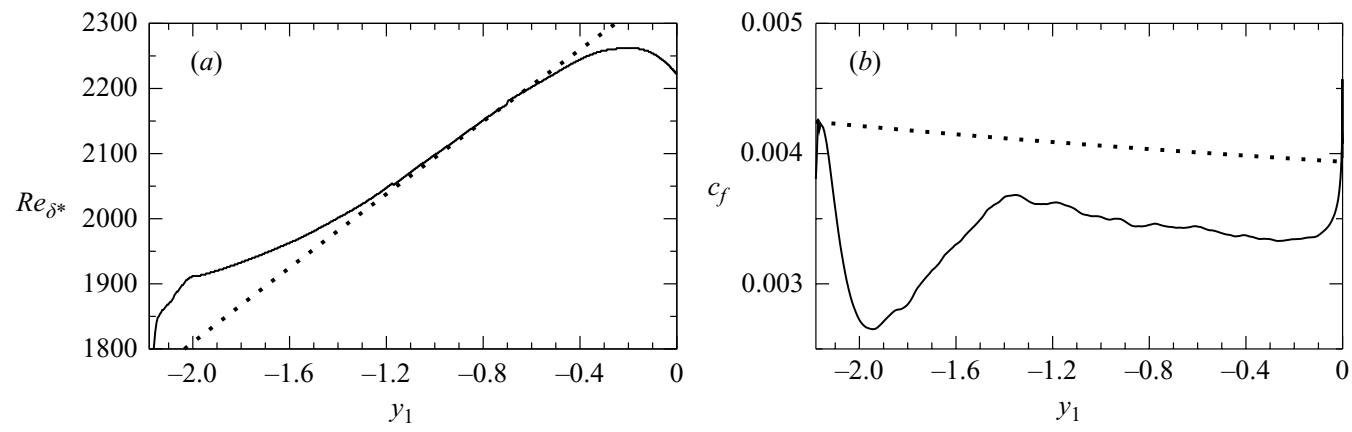

FiguRE 2. (a) Time-averaged Reynolds number based on displacement thickness and $(b)$ skin friction, on the top surface; the dotted lines denote values deduced from the $1 / 7$ th power velocity distribution law (Schlichting 1979) with the virtual origin at $y_{1}=-6.98$.

The boundary layer on the top side appears to be fully turbulent while the bottom boundary layer is laminar. The two boundary layers separate at the trailing edge and a turbulent wake develops downstream.

In order to evaluate whether the turbulent boundary layer is adequately resolved by the grid, the grid spacing is expressed in wall units. The first grid point away from the wall is located at $y_{2}^{+} \leqslant 0.8$, resulting in twelve points being clustered within the first $y_{2}^{+}=10$, and approximately 115 points resolving the boundary layer at the trailing edge. The spanwise grid spacing varies between $7 \leqslant \Delta y_{3}^{+} \leqslant 7.5$ over the plate. In the streamwise direction, the grid is strongly stretched. At $y_{1}=-0.727, \Delta y_{1}^{+}=14.95$ while at the trailing edge, $\Delta y_{1}^{+}=3.25$. In the streamwise and wall-normal directions, the grid resolution is finer than that employed in simulations of turbulent plane 
Simulation

Current

Sandham et al. (2002)
$\Delta y_{1}^{+} \quad y_{2}^{+}<10$

3.25

15
$\Delta y_{3}^{+}$

$12 \quad 7.5$

$10 \quad 7.5$

TABLE 2. Grid resolution in wall units at the trailing edge.
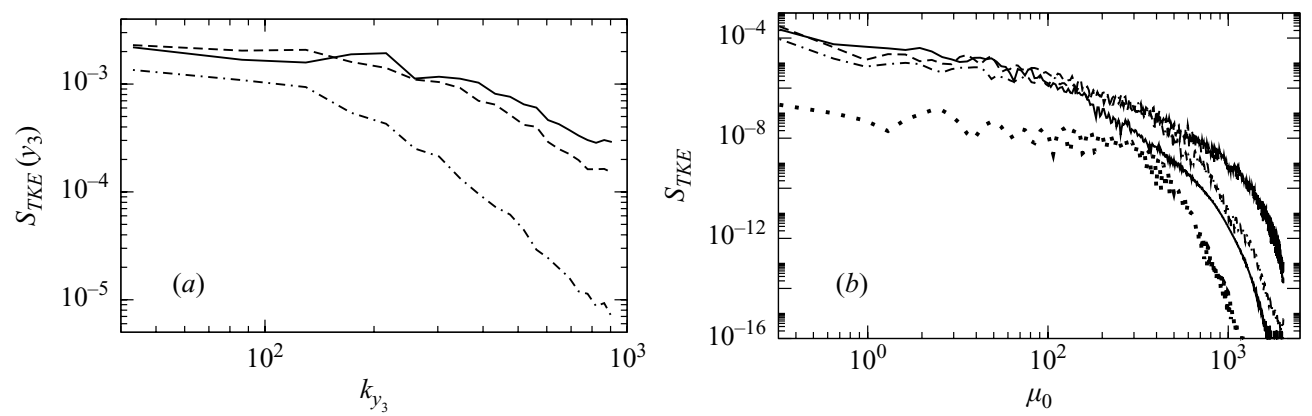

FIGURE 3. Power spectra of turbulent kinetic energy. (a) Spanwise spectrum, and (b) frequency spectrum:,$- y_{2}^{+}=12 ;---, y_{2}^{+}=50 ;-\cdot-, y_{2}^{+}=200 ; \cdots, y_{2}^{+}=600$.

channel flow with the same numerical method (Sandham et al. 2002) as illustrated in table 2. Nevertheless, the spanwise direction with a grid spacing of $\Delta y_{3}^{+} \approx 7.5$ is presumably the least well-resolved direction. For that reason, spectra of turbulent kinetic energy (TKE) in the boundary layer were scrutinized. Spanwise power point spectra of TKE were generated for several wall-normal distances from the DNS data, shown in figure 3(a). The spectra show a decay of at least one decade, depending on the distance from the wall. It should be noted however, that the time-dependent data used to generate the spectra were only written to file for every second spanwise point; hence the drop in energy will be greater than that illustrated. In addition, the frequency power spectra of TKE are presented in figure $3(b)$, showing a drop of at least ten decades for all locations in the wall-normal direction, implying that the temporal resolution is sufficient.

To assess the quality of the turbulent boundary layer, figure 4 shows the streamwise velocity and turbulent intensities $\left\langle u_{i}^{\prime} u_{j}^{\prime}\right\rangle$ in wall units, compared to benchmark data of an incompressible turbulent boundary layer over a flat plate at $R e_{\delta_{1}}=2000$ (Spalart 1988). At two locations upstream of the trailing edge, the streamwise velocity profiles in wall units agree well with the reference solution up to $y_{2}^{+} \approx 200$. In the outer layer, the profiles show a significant wake region and the maximum value of $u_{1}^{+}$is higher than that of the reference data, which cannot be solely attributed to the higher $R e_{\delta_{1}}$. The profile is reminiscent of a turbulent boundary layer subject to a weak adverse pressure gradient although no significant adverse pressure gradient is present in the streamwise direction.

Looking at the turbulent intensities, it can be observed that at both upstream positions, the turbulent boundary layer is not as 'full' as the reference case, although it should be noted that this was not the case when scaling the profiles with boundary layer thickness and not in wall coordinates. The amplitude of the streamwise velocity fluctuations and the shear stress component agree well with the reference data. However, the peak levels of the spanwise velocity fluctuations were significantly lower than in the DNS of Spalart. To investigate the reasons for the discrepancies with the 

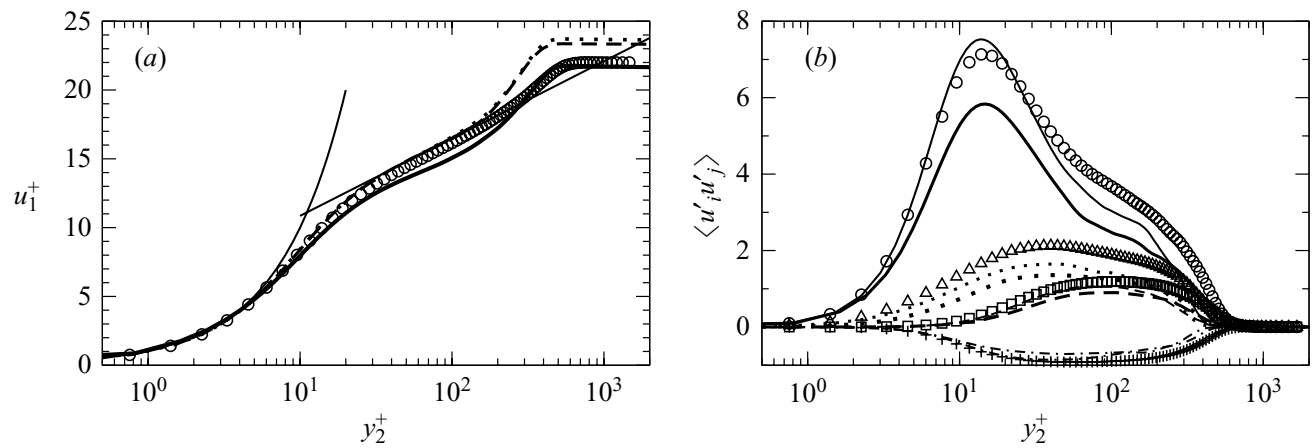

FiguRE 4. (a) Streamwise velocity profiles and $(b)$ turbulent intensities $\left\langle u_{i}^{\prime} u_{j}^{\prime}\right\rangle$, in wall units; in (a) circles denote data from incompressible reference DNS (Spalart 1988), thin lines represent $u_{1}^{+}=y_{2}^{+}$and $u_{1}^{+}=(1 / 0.41) \log y_{2}^{+}+5.24 ; \cdots$, DNS at $y_{1}=-0.727,-\cdots$, DNS at $y_{1}=-0.363$; thick solid line represents DNS at trailing edge; in $(b)$ symbols denote results from incompressible reference DNS (Spalart 1988), thin lines denote DNS results at $y_{1}=-0.363$; thick lines denote DNS results at the trailing edge, with $\bigcirc,-,\left\langle u_{1}^{\prime} u_{1}^{\prime}\right\rangle ; \square,--,\left\langle u_{2}^{\prime} u_{2}^{\prime}\right\rangle ; \Delta, \cdots$, $\left\langle u_{3}^{\prime} u_{3}^{\prime}\right\rangle ;+,-\cdot-,\left\langle u_{1}^{\prime} u_{2}^{\prime}\right\rangle$.

reference data, additional simulations of turbulent boundary layers (without trailing edge) were conducted using large-eddy simulations to reduce the computational cost. When omitting the inflow sponge, which led to unacceptable levels of noise caused by the turbulent inflow generation, the maximum $u_{1}^{+}$was reduced and approached the value of the reference data. The profiles also became significantly fuller and the amplitude of $\left\langle u_{3}^{\prime} u_{3}^{\prime}\right\rangle$ was increased.

Therefore, it appears that the long transition region (see figures 1 and 4), due to the inflow sponge, is responsible for the differences between the current DNS and the reference data. The turbulent boundary layer requires a long streamwise distance to fully develop. This is confirmed by looking at the profiles at the trailing edge. The turbulent intensity profiles are fuller than further upstream. As will be discussed below, the skin friction increases significantly towards the trailing edge, leading to a smaller maximum value of $u_{1}^{+}$and peak levels of the turbulent intensities.

Another measure for determining whether the turbulence is developed is to look at the spanwise correlation coefficient $\mathscr{R}_{p}$ of the disturbance pressure on the top surface, computed as

$$
\mathscr{R}_{p}\left(y_{1}, \Delta_{3}\right)=\left\langle\frac{\overline{p\left(y_{1}, y_{3}, t\right) p\left(y_{1}, y_{3}+\Delta_{3}, t\right)}}{\overline{p^{2}\left(y_{1}, y_{3}, t\right)}}\right\rangle,
$$

where the overbar denotes a time average and \langle\rangle is the spanwise average. The distribution of $\mathscr{R}_{p}$ for several streamwise locations is shown in figure 5 . Within the sponge region (not shown) a high spanwise correlation can be observed, gradually decreasing in the downstream direction. At the trailing edge, $\mathscr{R}_{p}$ drops to values of less than 0.1 for all but very small and large spanwise distances $\Delta_{3}$. The high level of spanwise correlation for the smallest and largest values of $\Delta_{3}$ is due to the use of periodic boundary conditions.

Even though the turbulence might not be fully developed upstream of the trailing edge, it is probably representative of the flow over the trailing edge of an airfoil at moderate Reynolds number. In that case, laminar-turbulent transition takes place a considerable distance from the leading edge and therefore the turbulent boundary layer convecting past the trailing edge most likely is also not fully developed. Overall, 


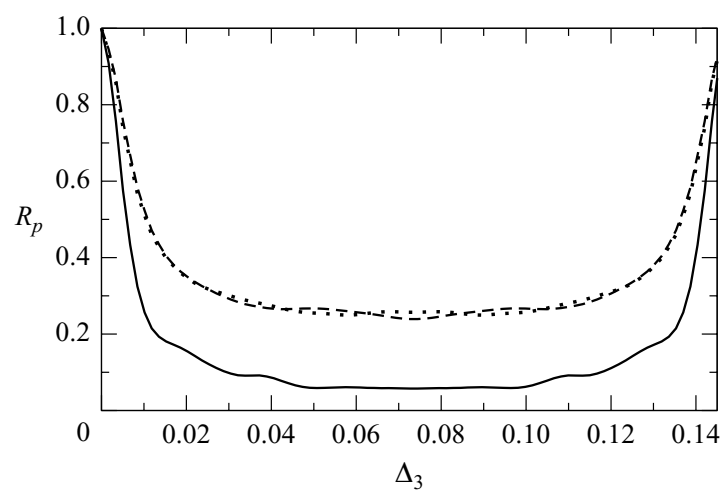

FIGURE 5. Spanwise correlation of pressure on the top surface in the time domain: $\cdots$, $y_{1}=-0.727 ;---, y_{1}=-0.363 ;-$, , trailing edge.

in spite of some differences from the benchmark data, the simulation succeeds in producing a turbulent boundary layer passing over an infinitely thin trailing edge. Thus, the DNS data will be used to investigate the behaviour of a turbulent boundary layer in the vicinity of a trailing edge in the following section.

As could be seen from figure 2, on approaching the trailing edge the displacement thickness decreases and the skin friction rises sharply, consistent with analytic predictions using triple-deck theory (cf. Stewartson 1968; Messiter 1970; Daniels 1977; Gajjar \& Türkyilmazog̃lu 2000). However, the asymptotic solutions provided in the literature are derived for laminar boundary layers. Here, the applicability of the results of Stewartson (1968) and Messiter (1970) for turbulent flow is evaluated. According to the triple-deck structure, for a flat plate of length $l$ the boundary layer solutions (where the boundary layer thickness scales with $l R e_{l}^{-1 / 2}$ ) lose their validity within a distance of $O\left(l R e_{l}^{-3 / 4}\right)$ from the trailing edge. Stewartson (1968) derived analytic expressions for the skin friction in the immediate vicinity of the trailing edge

$$
c_{f}\left(y_{1}\right)=\frac{4 \lambda}{1.0651^{3 / 4} R e_{l}^{1 / 2}}\left[\left(\frac{2 l}{-\pi \lambda^{1 / 2} R e_{l}^{3 / 4} y_{1}}\right)^{1 / 2}+0.39\left(\frac{-\lambda^{1 / 2} R e_{l}^{3 / 4} y_{1}}{\pi 2 l}\right)^{1 / 2}\right],
$$

and at a greater distance from the trailing edge

$$
c_{f}\left(y_{1}\right)=\frac{2 \lambda}{R e_{l}^{1 / 2}}\left[1+\frac{0.8966 \Gamma(-1 / 3) \sqrt{3}}{2 \pi}\left(\frac{2 l}{-\lambda^{1 / 2} R e_{l}^{3 / 4} y_{1}}\right)^{2 / 3}\right],
$$

where $\lambda=0.332$. Equations (5.2) and (5.3) are referred to as the 'inner' and 'outer' solution, respectively, in the following. As seen in figure 2 the theoretical prediction of skin friction for a flat-plate boundary layer without trailing edge produces larger values than found in the current DNS. This is most likely due to the influence of the inflow sponge layer, effectively moving the virtual origin farther upstream. To account for the difference, the skin friction coefficients computed from (5.2) and (5.3) are shifted by a small correction factor compared with the DNS data. Also, a curve fit of the DNS data in the interval $-1.2 \leqslant y_{1} \leqslant-0.3$ is generated that is assumed to be the skin friction if no trailing edge were present.

In figure 6 the skin friction coefficient obtained from DNS is compared to the analytic prediction of Stewartson (1968). The outer solution predicts an increase in $c_{f}$ 

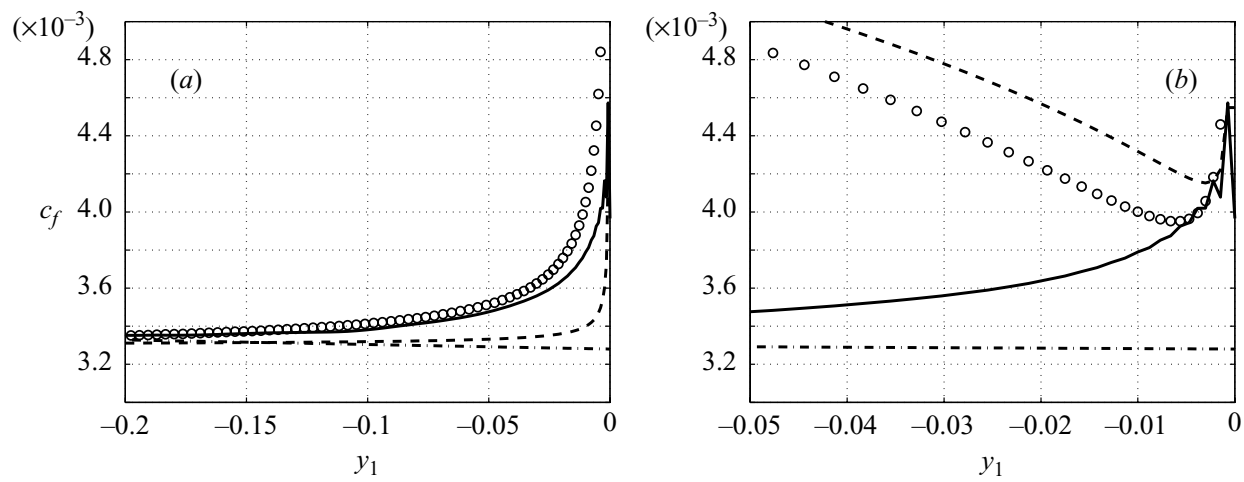

FiguRE 6. Time averaged skin friction coefficient $c_{f}$ on top surface for $(a)$ the outer and $(b)$ inner region:- DNS; $-\cdot-\cdot-$, curve fit of DNS data excluding data for $y_{1}>-0.3 ;-\cdots$, solution according to Stewartson (1968); O, solution according to Stewartson (1968) but using the eddy viscosity.

when approaching the trailing edge. However, the agreement with DNS data is poor in terms of the onset of the increase and the amplitude reached at the trailing edge. This is not surprising as (5.2) only strictly should apply to laminar flows. To account for the turbulent nature of the present boundary layer, a simple correction is made. The eddy viscosity $v_{T}$ of the turbulent boundary layer is estimated using Prandtl's mixing length theory (see Schlichting 1979). For the logarithmic region the eddy viscosity can be estimated as $v_{T}=\kappa y_{2} u_{\tau}$, with $\kappa=0.41$. As the triple-deck solution is only valid very close to the wall, the eddy viscosity was evaluated at $y_{2}^{+}=20$ and assumed to be constant in the wall-normal direction. To account for the turbulent viscosity in (5.2) and (5.3), the Reynolds number based on chord $R e_{l}$ is divided by the ratio of eddy viscosity over molecular viscosity, which was 8 for the present case. Using the reduced effective Reynolds number, (5.2) and (5.3) are used again to compute the skin friction for the turbulent boundary layer. Good agreement with the DNS data is achieved for the outer solution for both the onset of the departure from the 'no trailing edge' skin friction, and for the initial slope. The slope obtained from the inner solution in the immediate vicinity of the trailing edge also compares favourably with the DNS data. The reduced effective Reynolds number leads to the inner region following the DNS data for a larger streamwise extent, suggesting that the numerical resolution of this region is just sufficient. The inner solution of the triple-deck analysis for laminar flow, on the other hand, would not have been captured in the streamwise direction with the current DNS grid because the sharp increase in skin friction would have been confined to a considerably smaller streamwise extent.

Stewartson (1968) also provides analytic solutions for the streamwise mean velocity downstream of the trailing edge on the centreline, $y_{2}=0$. For the region within $l R e_{l}^{-3 / 4}$ of the trailing edge, the streamwise velocity component scales as $u\left(y_{1}\right)=R e_{l}^{1 / 8}\left(y_{1} / 2\right)^{1 / 2}$ while for $y_{1}>l R e_{l}^{-3 / 4}$ the asymptotic solution is $u\left(y_{1}\right)=\left(y_{1} / 2\right)^{1 / 3}\left[1-R e_{l}^{-1 / 2}\left(y_{1} / 2\right)^{-2 / 3}\right]+c$. The resulting streamwise velocity distribution on the centreline is shown in figure 7 and compared with the DNS data. The dashed line represents the asymptotic solutions if the eddy viscosity is neglected. When the turbulent viscosity is accounted for using the same scaling factor as for the evaluation of the skin friction, the inner solution follows the DNS data up to a streamwise position of $y_{1}=0.005$ and the outer solution approaches the DNS data for $y_{1}>0.12$. 


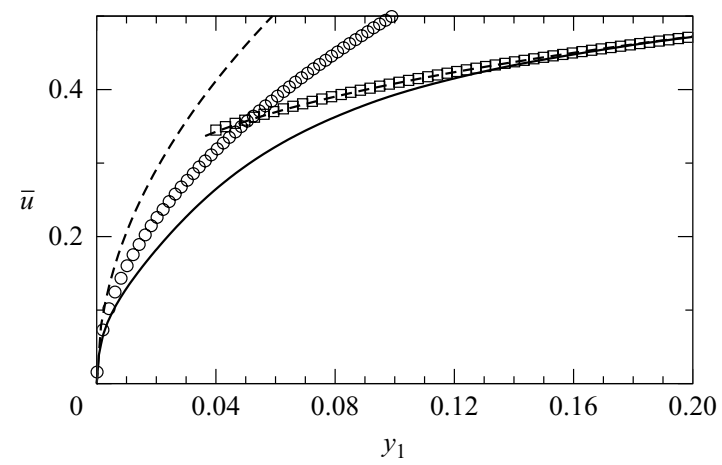

FIGURE 7. Streamwise mean velocity downstream of the trailing edge on the centreline, $y_{2}=0$ : $\longrightarrow$ DNS; - - -, laminar solutions according to Stewartson (1968); $\bigcirc, \square$, inner and outer solution, respectively, according to Stewartson (1968) but using the eddy viscosity.

Good agreement over a wider range of $y_{1}$ can be achieved by increasing the ratio between eddy and molecular viscosity to 40, indicating that using the eddy viscosity evaluated at a single point in the turbulent boundary layer might not be appropriate. For the current case, the wake velocity constitutes a difficult problem, as it depends on both the turbulent and laminar streams on the top and bottom side of the plate, respectively; thus the choice of the correct length scale is not clear. Therefore, further refinement of the current approach is necessary.

The behaviour of the streamwise velocity component downstream of the trailing edge also provides an intuitive explanation for the behaviour of the skin friction and the displacement thickness upstream of the trailing edge. The velocity increases monotonically in the streamwise direction, thus a positive streamwise gradient of the streamwise velocity component is present. Therefore, from the continuity equation, when neglecting the small variation in density and spanwise velocity, the wall-normal gradient of the wall-normal velocity component needs to be negative, implying a negative wall-normal velocity component for $y_{2}>0$ due to the no-penetration condition on the surface. This inevitably causes a thinning of the boundary layer and a subsequent increase in the wall shear stress. Note that for many airfoil flows, a small recirculation region exists downstream of the trailing edge, resulting in the opposite behaviour in the vicinity of the trailing edge, i.e. an increase in displacement thickness associated with a decrease in skin friction. This is confirmed in Gajjar \& Türkyilmazog̃lu (2000) who solved the triple-deck equations for airfoils with wedged trailing edges.

According to Messiter (1970) the pressure in the vicinity of the trailing edge can be approximated as

$$
p\left(y_{1}\right)= \begin{cases}\frac{1}{\gamma M^{2}}\left(1-\frac{c_{1}}{3 \sqrt{3} \sqrt{0.332}} R e_{l}^{-1 / 2}\left(-y_{1} / 2\right)^{-2 / 3}\right), & y_{1}<0 \\ \frac{1}{\gamma M^{2}}\left(1-\frac{c_{1}}{3 \sqrt{3} \sqrt{0.332}} R e_{l}^{-1 / 2}\left(y_{1} / 2\right)^{-2 / 3}\right), & y_{1}>0,\end{cases}
$$

Figure $8(a)$ shows the asymptotic solution of the mean pressure compared with the DNS result. Note that the Reynolds number was scaled with the same factor as used for the analysis of the skin friction coefficient to account for the turbulent viscosity. The mean pressure decays towards the trailing edge, resulting in a favourable pressure gradient on the surface. The behaviour of the pressure fluctuations in the vicinity 

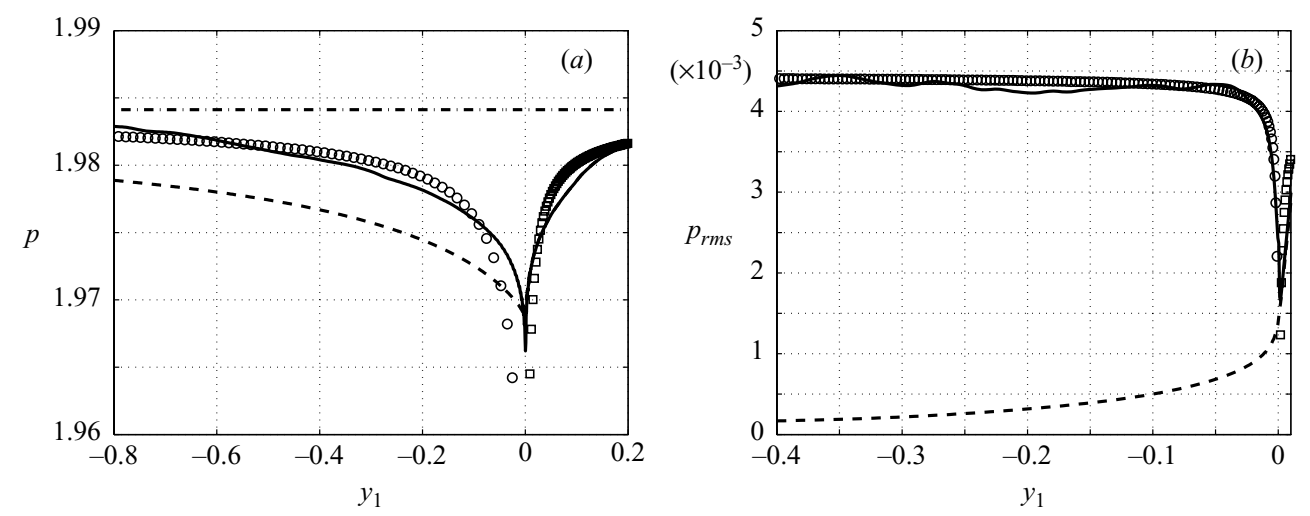

Figure 8. (a) Mean pressure and $(b) p_{\text {rms }}$ on the surface: - - top surface; - - , bottom surface; $-\cdot-\cdot-, p=1 /\left(\gamma M^{2}\right)$, symbols denote solutions according to Messiter (1970) but with eddy viscosity.
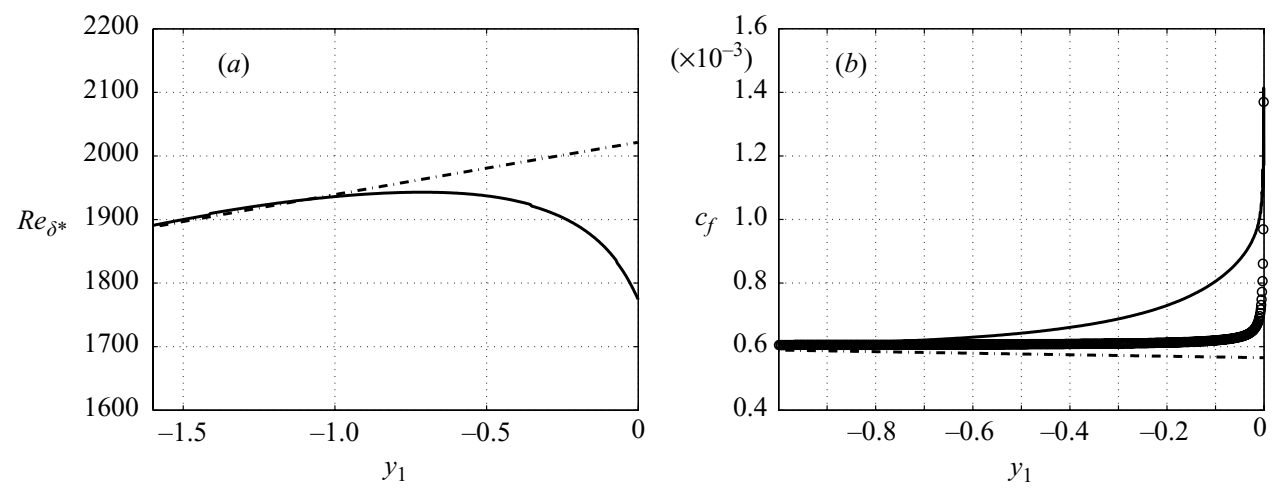

FIGURE 9. (a) Time-averaged Reynolds number based on displacement thickness and $(b)$ skin friction coefficient $c_{f}$ : $\longrightarrow$ DNS; $-\cdot-\cdot-$, laminar solutions from Schlichting (1979); $\bigcirc$, laminar solution according to Stewartson (1968) ( $b$ only); virtual origin at $y_{1}=-16$.

of the trailing edge is of great interest because the sound radiation is proportional to the disturbance pressure amplitudes in that region. The value of $p_{r m s}$ is shown in figure $8(b)$ for the top and bottom surfaces. On the top surface, $p_{r m s}$ decays rapidly towards the trailing edge whereas the converse occurs on the bottom surface. This is due to the fact that no disturbances were introduced on the lower side and all surface pressure fluctuations are due to the scattered pressure field, which decays in the upstream direction. The mean pressure value $\left(1 / \gamma M^{2}\right)$ in (5.4) was replaced by the mean value of $p_{r m s}$ upstream of the trailing edge in an attempt to determine whether the asymptotic solution also applies to the pressure fluctuations. The solution obtained shows very good agreement with the DNS data, as shown in figure $8(b)$.

Conducting the simulation with a turbulent boundary layer on the top side only also provides the opportunity to investigate the behaviour of the lower-surface laminar boundary layer in the vicinity of a trailing edge. Figure 9 shows the Reynolds number based on displacement thickness and the skin friction obtained from DNS compared with laminar solutions from Schlichting (1979) and Stewartson (1968). The Reynolds number based on displacement thickness starts to deviate from the laminar solution for a boundary layer without a trailing edge starting at $y_{1}=-1$, in contrast to the 

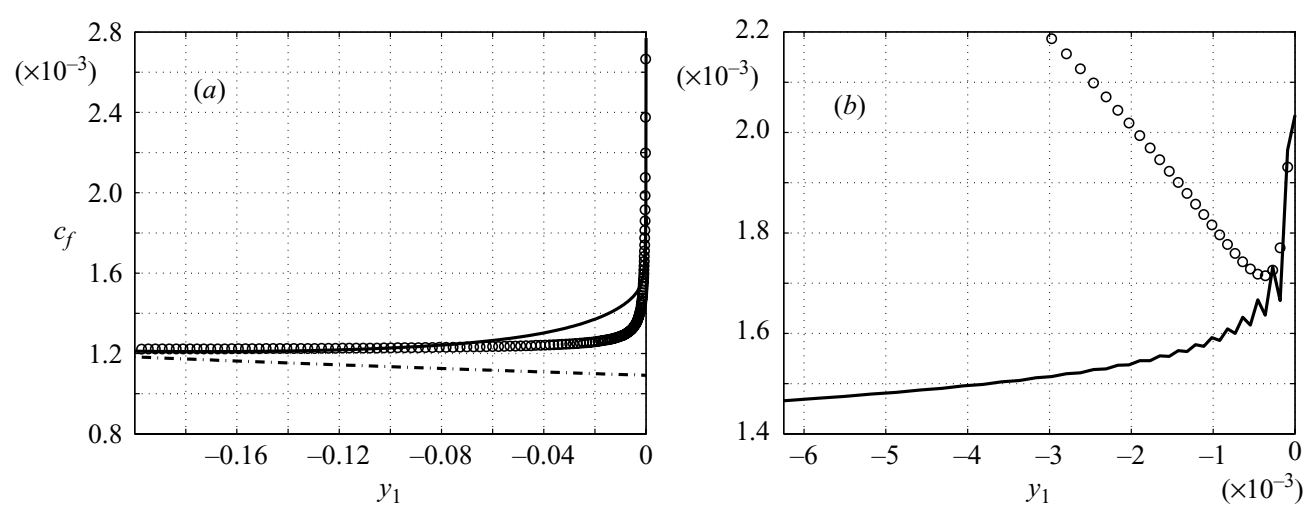

FIGURE 10. Time-averaged skin friction coefficient from two-dimensional laminar resolution study for $(a)$ outer and $(b)$ inner region; _ (1979); $\bigcirc$, laminar solution according to Stewartson (1968); virtual origin at $y_{1}=-16$.

turbulent case (figure $2 a$ ), where the first deviation from the approximation for a turbulent boundary layer by Schlichting (1979) occurs at $y_{1}=-0.5$. The skin friction coefficient departs the laminar solution at $y_{1}=-0.6$, while in the turbulent case, shown in figure $6 a), c_{f}$ follows the 'no trailing edge' solution up to approximately $y_{1}=-0.2$. The analytic result of Stewartson (1968), however, predicts a sharp rise in skin friction confined to the immediate neighbourhood of the trailing edge. The disagreement between the DNS data and the theory is due to the pressure field being strongly modified by the Reynolds stress components of the turbulent boundary layer on the top side.

In order to verify that the discrepancy between the laminar side of the DNS and the analytic solution is due to the effect of the top-side turbulent boundary layer on the pressure field, the data from a precursor resolution study were also compared with the triple-deck solution of Stewartson (1968). In the two-dimensional laminar simulations, the boundary layers were specified to be equal on the top and bottom side, thus yielding a symmetric solution. Figure 10 shows the time-averaged skin friction coefficient obtained from the two-dimensional laminar simulations compared with the analytic solutions of Stewartson (1968) for both large and small distances from the trailing edge. The solution for large $y_{1}$ agrees with the DNS data up to $y_{1}=-0.08$, while the slope determined from the triple-deck theory for small $y_{1}$ is similar to the slope obtained from DNS for $y_{1}>-2 \times 10^{-4}$. Thus the behaviour of the laminar flow in the vicinity of the trailing edge can be predicted fairly accurately, confirming that the differences found between the lower-side boundary layer of the three-dimensional simulation and the theory are due to the influence of the top-side turbulent boundary layer.

Overall, the above results imply that the scalings derived by Stewartson (1968) and Messiter (1970) produce results with reasonable accuracy for turbulent boundary layers when the eddy viscosity of the turbulence is accounted for. Furthermore, good agreement can be achieved using a crude approximation for $\nu_{T}$, although the results are sensitive to the value of $v_{T}$.

\subsection{Acoustic field}

The acoustic pressure can be obtained directly from the DNS. In addition, the twoand three-dimensional acoustic analogies can be used to predict the acoustic pressure as a function of the total surface pressure difference computed using DNS. Finally, 

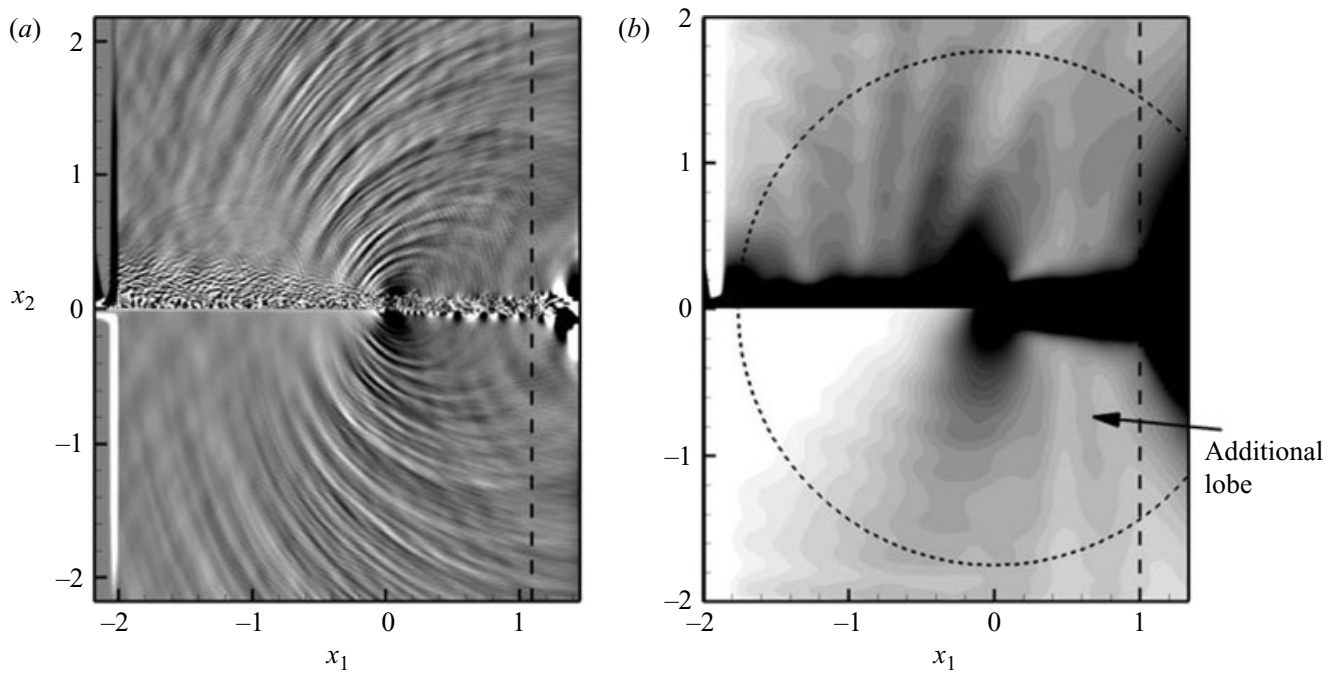

FIGURE 11. (a) Instantaneous contours of dilatation $\left[2 \times 10^{-3} ; 2 \times 10^{-3}\right]$, $(b)$ contours of $p_{\text {rms }}$ $\left[1 \times 10^{-4} ; 4 \times 10^{-4}\right]$ with dashed circle showing $R_{d}=1.75$, the vertical dashed line denotes the onset of the zonal characteristic boundary condition; $x_{3}=0.0725$.

the classical theory of Amiet is employed to predict the far-field pressure and the assumptions invoked in the theory are scrutinized using the DNS data.

\subsubsection{DNS results}

One of the main objectives of the current research is to investigate the broadband trailing-edge noise generation. It is therefore essential that noise generated at the trailing edge is represented accurately by the simulation. Specifically, reflections from the boundaries and noise generated by the turbulent inflow generation technique must not interfere with, or even be larger than, the trailing-edge noise. Instantaneous contours of dilatation for $y_{3}=0.725$, shown in figure $11(a)$, illustrate that the trailing edge is the main source of noise. The inflow sponge appears to be successful in attenuating most of the noise generated by the turbulent inflow generation and the zonal characteristic boundary condition is able to sufficiently reduce reflections due to the wake structures passing the outflow boundary. The vertical dashed line marks the onset of the zonal characteristic boundary condition. The turbulent boundary layer itself appears to be a source of noise, with the resulting acoustic waves interacting with the trailing-edge noise. On the bottom side, only the acoustic waves generated at the trailing edge are present and can therefore be observed clearly, a key advantage of conducting DNS with a turbulent boundary layer on one side of the surface only.

This becomes even clearer when scrutinizing contours of $p_{\text {rms }}$ (figure $11 b$ ) at $y_{3}=0.725$ obtained by averaging over more than two flow-through times. It is evident that the top side is composed of more than one noise source while trailing-edge noise is the dominant component on the bottom side. Another observation that can be made when examining the contours of $p_{r m s}$ is the presence of an additional 'lobe' on the bottom side that does not have its origin at the trailing edge but some distance into the wake. This additional lobe is an indication that the nonlinear wake source mechanism described in Sandberg et al. (2007) might also apply to the fully turbulent case. As it contains radiation for all frequencies $p_{r m s}$ is therefore not very useful for 

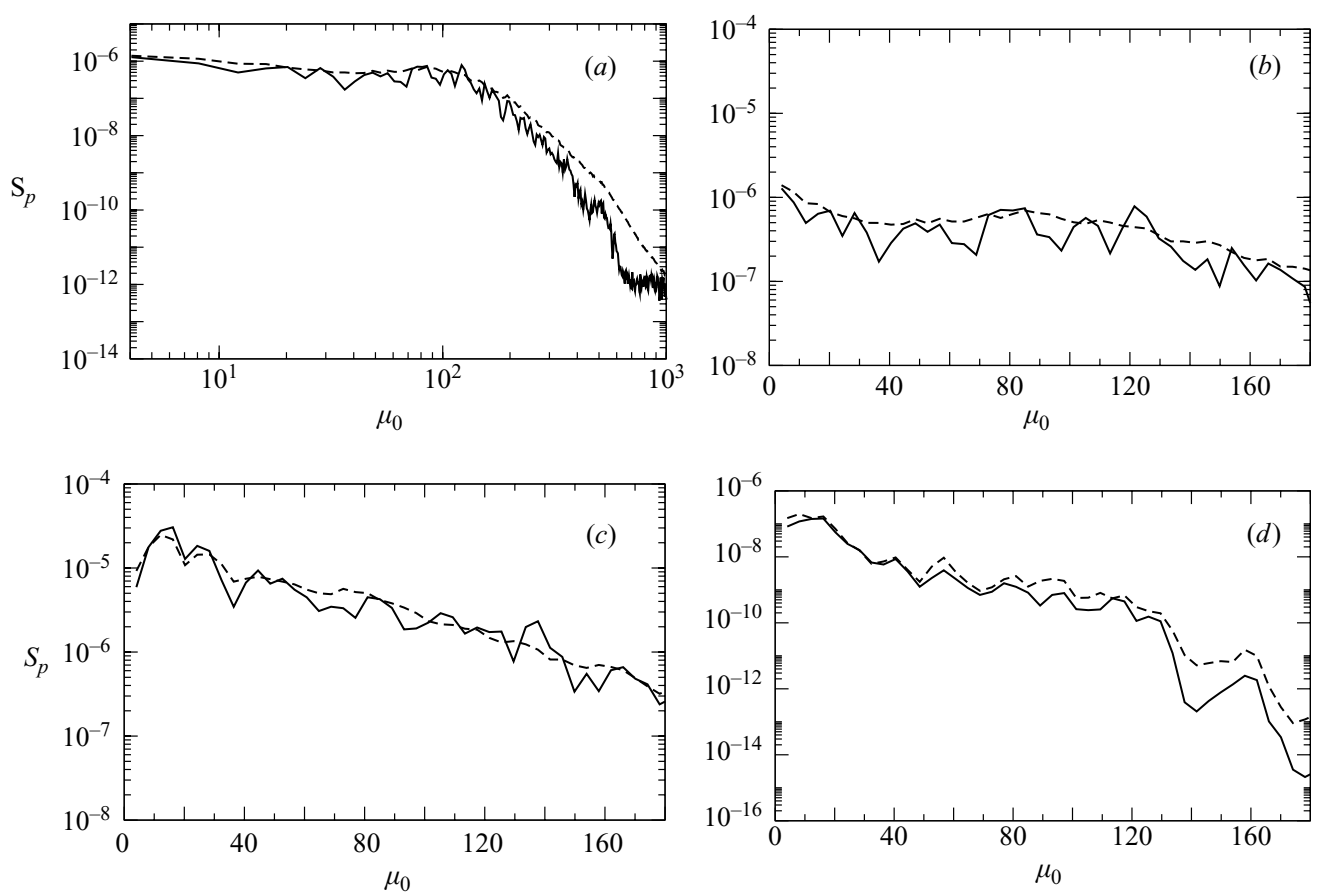

FIGURE 12. $(a-c)$ Point spectra of surface pressure difference: - , data at the lateral plane $y_{3}=0.0725$; - - - , span-averaged data; and $(d)$ point spectra of pressure in the radiation field from DNS: — and averaging over seven segments. $(a, b) y_{1}=0,(c, d) y_{1}=-0.363$.

further analysis. Instead, the DNS data are Fourier transformed in time to further investigate the trailing-edge noise in frequency space.

The record length of the original time series is $\tau=40$ with a sampling interval of $\Delta t=0.008$. To obtain smoother spectra, the procedure suggested by Hu, Morfey \& Sandham (2006) was adopted. The time record is split into seven segments with a $50 \%$ overlap. The time average of each segment is subtracted from the signal before applying a Hanning window. The modified data are then Fourier transformed in time and an average over all segments and spanwise locations is taken. The frequency spectrum $S_{p}$ is then obtained by multiplying the point spectrum with its complex conjugate, dividing by the record length $\tau$, and applying the factor $8 / 3$ to account for the windowing. Figure 12(a-c) shows point spectra of surface pressure difference obtained from data at $y_{3}=0.0725$ and span-averaged data. The spectra at the trailing edge (figure $12 a$ ) exhibit a fairly broad band of frequencies up to $\mu_{0} \approx 200$ with large amplitudes. For higher frequencies, the amplitudes decrease by nearly six decades, implying that the temporal resolution of the DNS is sufficient to capture the smallest time scales. Only the low-frequency region of the spectra at the trailing edge and upstream of the trailing edge are shown in figures $12 b$ ) and $12 c$ ), respectively. When the data from the lateral plane at $y_{3}=0.725$ were analysed, distinct peaks at several frequencies could be observed. When using the spanwise-averaged data, the spectra were significantly smoother and no dominant frequencies were present, except at very low frequencies. It is important to note that the amplitudes of the spectra at the trailing edge are considerably lower than at the upstream location, in particular for very low frequencies, a phenomenon not observed in preliminary simulations of 
Inflow forcing frequencies

\begin{tabular}{lll}
\cline { 2 - 3 }$\mu_{0}$ & 3.2 & 6.4 \\
Periods available & 4 & 8
\end{tabular}

Frequencies not forced

\begin{tabular}{llll}
\hline 16.2 & 26.3 & 40.5 & 85.0 \\
20 & 32 & 50 & 105
\end{tabular}

TABLE 3. Selected reduced frequencies appearing in all spectra.

airfoil trailing edges and the experiments by Brooks \& Hodgson (1981). It is therefore suggested that this is due to the rapid increase in the skin friction coefficient and the thinning of the boundary layer. As mentioned before, the thinning of the boundary layer towards the separation point and the resulting increase in skin friction is only observed in the case of a flat-plate trailing edge. The associated reduction of the surface pressure difference amplitude at lower frequencies is therefore most likely restricted to this particular geometry. This circumstance suggests that trailing edge noise reduction might be achieved on the basis of modifying the airfoil trailing-edge geometry. It should also be noted that a streamwise change of the spectra is in contradiction with Amiet's assumption of 'frozen turbulence'.

In figure $12(d)$ point spectra of pressure are shown for points on the top and bottom side of the radiation field obtained from data of the lateral plane at $y_{3}=0.725$. At lower frequencies, the spectra are very similar, showing roughly the same amplitudes and peaks for the same frequencies. For higher frequencies the peak locations remain similar but the spectrum computed from data on the top side of the radiation field exhibits larger amplitudes. This is most likely due to the trailing edge noise being superposed with noise produced by the boundary layer and the inflow turbulence generation.

In order to decide at which frequencies to investigate the trailing edge noise, spectra of surface pressure difference and in the radiation field were studied (figure $12 b-d$ ). A selection of reduced frequencies found to appear in all spectra is given in table 3, distinguishing between frequencies that were introduced through the turbulent inflow generation technique and frequencies that were not forced. In addition, the number of periods available for each frequency is given. The very low frequencies appearing in the spectra can be associated with the inflow forcing, and data were only available for a small number of periods. However, for all non-forced frequencies that were selected, data were available for a sufficient number of periods.

Because of the large number of points required for the DNS, it was impractical to store the entire time series of the full three-dimensional data. Instead, a significant number of two-dimensional surfaces was selected for which the entire time series was written to file. These surfaces include the lateral plane at $y_{3}=0.725$, planes parallel to the surface of the plate and several streamwise planes.

In figure 13, contours of the real part of pressure, with an inset of the spanwise vorticity component, are shown for several frequencies for the plane at $y_{3}=0.725$. The top two plots, $(a)$ and $(b)$, show the two lowest frequencies investigated, which are associated with the turbulence inflow generation. For both frequencies, on the bottom side sound waves originating from the trailing edge can be observed, whereas the origin of the sound waves on the top side is not evident, leading to asymmetric sound radiation. For all non-forced frequencies investigated, shown in figures $13(c)$ and $13(d)$ (and later in figure 15), the sound radiation clearly originates from the trailing edge and the top and bottom sides are nearly symmetric. This suggests that 
(a) $\mu_{0}=3.2,\left[-1 \times 10^{-4} ; 1 \times 10^{-4}\right]$

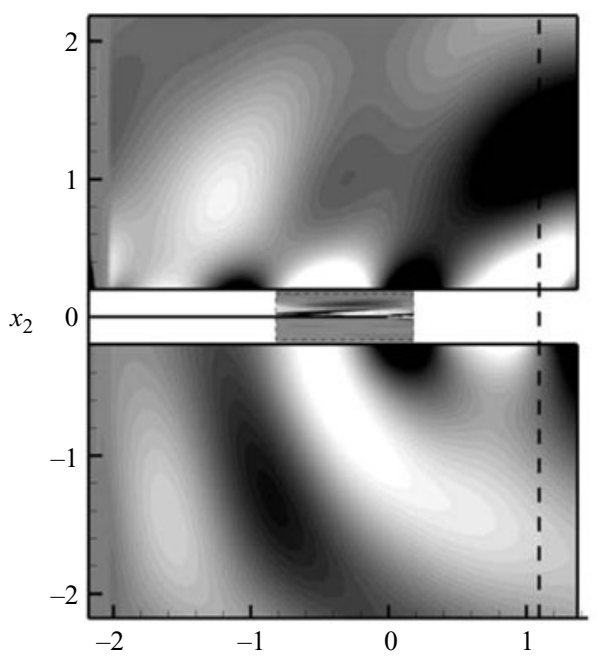

(c) $\mu_{0}=26.3,\left[-7 \times 10^{-5} ; 7 \times 10^{-5}\right]$

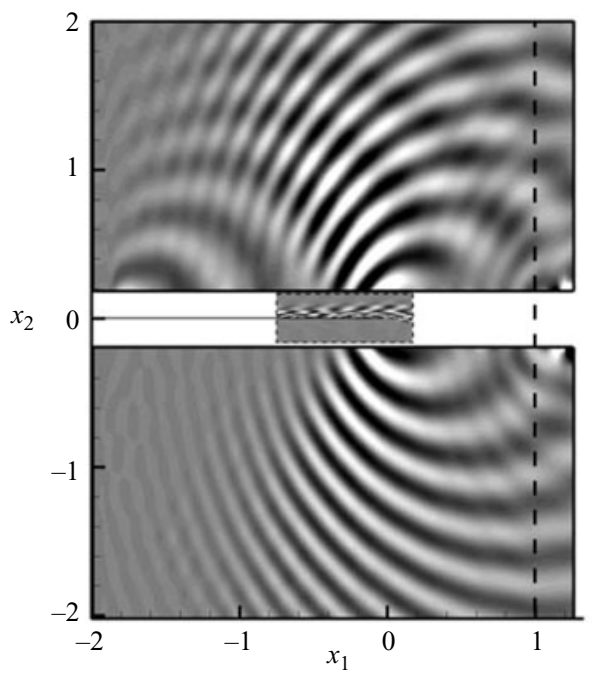

(b) $\mu_{0}=6.4,\left[-1 \times 10^{-4} ; 1 \times 10^{-4}\right]$

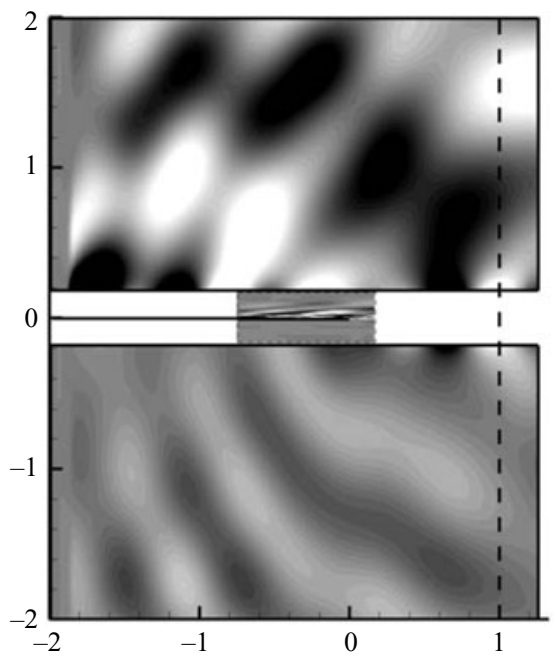

(d) $\mu_{0}=40.5,\left[-2 \times 10^{-5} ; 2 \times 10^{-5}\right]$

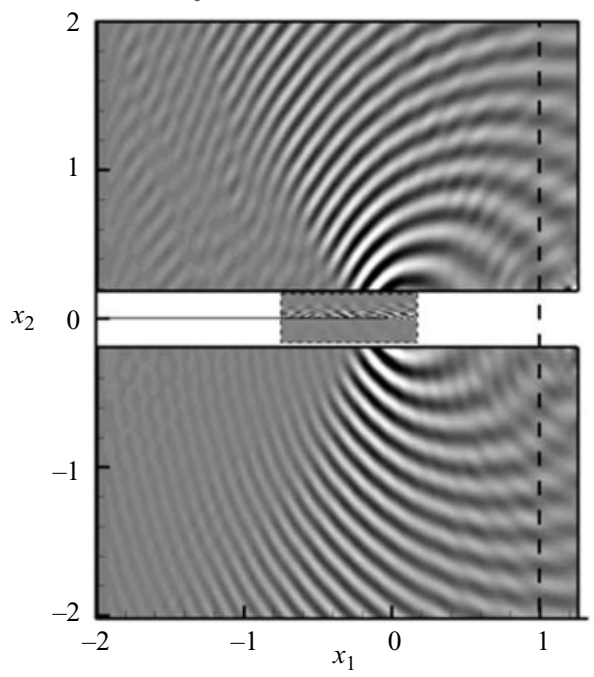

FIGURE 13. Real part of pressure and spanwise vorticity (inset) in the frequency domain for the lateral plane at $y_{3}=0.725$; range of vorticity $[-0.2 ; 0.2]$, range of the real part of pressure given for each individual frequency.

in spite of the presence of a sponge at the inflow, acoustic waves from the turbulent inflow generation propagate into the region of interest.

For the non-forced frequency $\mu_{0}=26.3$ an additional source of sound can be identified on the top surface at $x_{1} \approx-1.8$ It is conjectured that a vortex breakdown mechanism in the laminar-turbulent transition process of the boundary layer is responsible for this additional source. In general, the wavelength of the sound waves decreases considerably with increasing frequency, and the directivity changes. Looking at the spanwise vorticity component in frequency space, it can be observed that the streamwise spacing of oppositely signed vorticity for each frequency corresponds to half the acoustic wavelength. From figure 13 it is apparent that for the lowest 

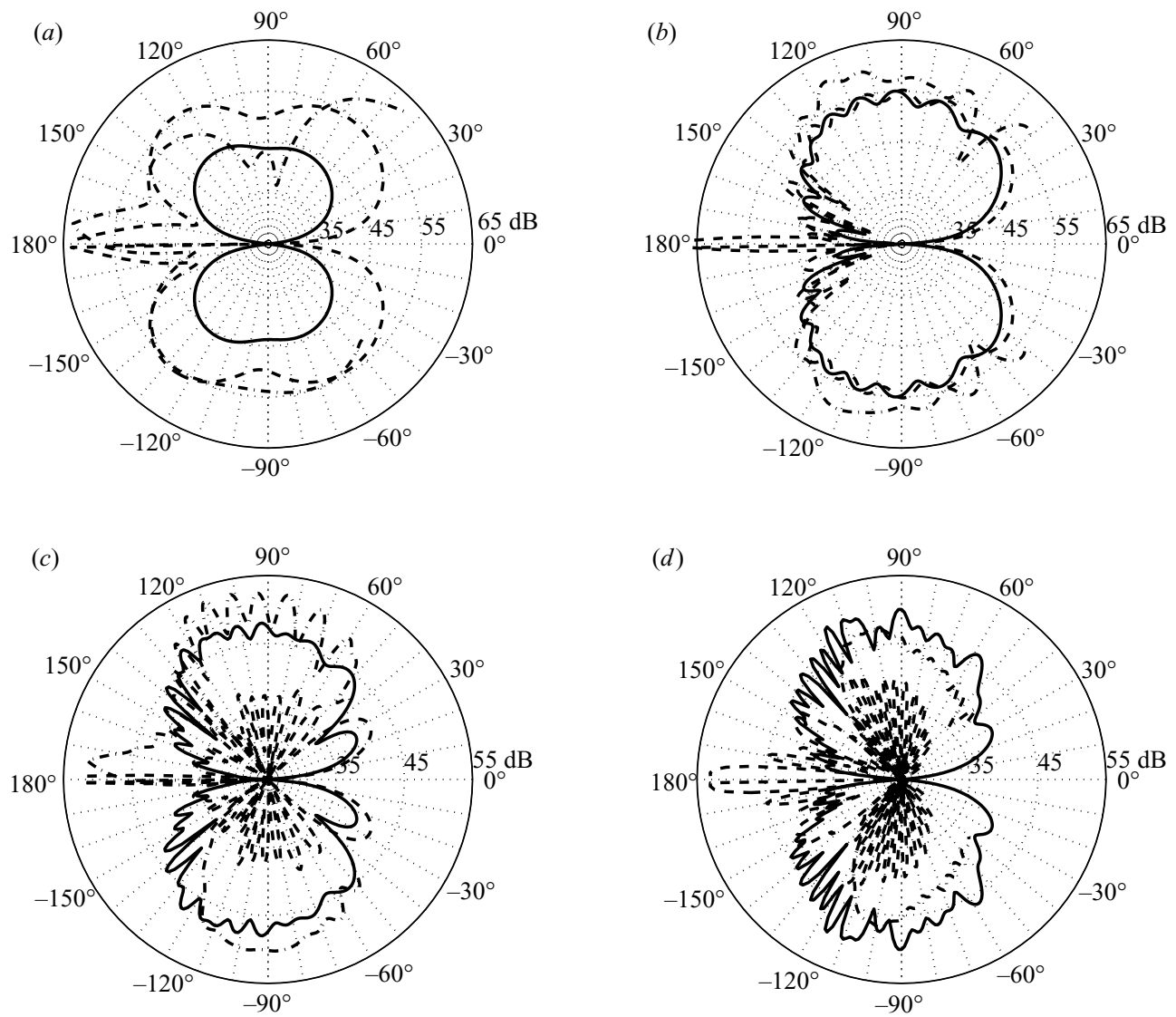

FIGURE 14. Magnitude of acoustic pressure in $\mathrm{dB}$ at the lateral plane $y_{3}=0.725 ;-,|p|$ using the three-dimensional acoustic analogy; - - -, $|p|$ using the two-dimensional acoustic analogy; $-\cdot-,|p|$ DNS; $R_{d}=1.75$. (a) $\mu_{0}=3.2,(b) \mu_{0}=16.2,(c) \mu_{0}=26.3,(d) \mu_{0}=40.5$.

frequencies investigated, a larger computational domain would have been beneficial in order to capture more than just one or two acoustic wavelengths. However, for the non-forced frequencies, which are of more interest, the size of the domain appears sufficient.

\subsubsection{Acoustic analogies}

The acoustic pressure is computed using the three-dimensional and two-dimensional acoustic analogies (equations (2.6) and (2.17)). The magnitude of the acoustic pressure evaluated using both methods is shown in directivity plots on figure 14 and compared to the acoustic pressure directly obtained from DNS at the radius $R_{d}=1.75$ from the trailing edge. Note that the DNS data are truncated for angles smaller than $\pm 30^{\circ}$ because the computational domain only extends to $x_{1}=1.45$. Nevertheless, this relatively large radius was chosen because it was found that near-field effects were significantly reduced compared to radii closer to the trailing edge. Furthermore, the grid at this radius is sufficiently fine that acoustic waves with the highest reduced frequency investigated here are resolved with 32,8 , and 64 points per wavelength in the streamwise, wall-normal, and spanwise directions, respectively. 
For both forcing frequencies and for $\mu_{0}=26.3$, where sound radiation originating from close to the inflow boundary on the top surface was observed in figure 13, noise sources on the top side in addition to the trailing edge noise lead to an asymmetric sound field as evidenced by the directivity patterns obtained directly from DNS. This cannot be accounted for by either form of the theory because the acoustic pressure is evaluated as a function of surface pressure difference and therefore inevitably produces a symmetric directivity distribution with respect to $x_{2}=0$. Nevertheless, on the bottom side of the plate only trailing edge noise is present and therefore a comparison between theoretical predictions and DNS data is still meaningful for frequencies with significantly asymmetric acoustic radiation fields.

Comparing the amplitudes obtained from both theories to the DNS data, the agreement is not as good as in the case of strictly two-dimensional simulations of disturbances over a trailing edge (Sandberg et al. 2007). This can probably be attributed to two factors. First, for the DNS, periodic boundary conditions in the spanwise direction are used, which is not accounted for in either theory. Each source on the surface of the plate radiates spherically, i.e. sound waves will eventually reach a lateral boundary. Instead of leaving the domain, the sound waves pass the boundary on one side and enter the domain on the other side, thus remaining within the computational domain. They can therefore interfere with the sound waves directly radiated. Depending on the phase information of the additional sound waves, the amplitude of the DNS data from the lateral plane at $y_{3}=0.725$ might decrease or increase. Secondly, in the present simulation the flow is turbulent and the turbulent viscosity is considerably larger than the molecular viscosity. Therefore, it can be conjectured that viscous effects, which did not appear to play a significant role in the two-dimensional laminar simulations, might be important.

For the lowest frequency investigated, $\mu_{0}=3.2$, it can be observed that the two- and three-dimensional acoustic analogy yield a similar directivity pattern. However, the amplitude predicted by the single-frequency two-dimensional theory is significantly higher and roughly corresponds to that obtained directly from DNS. This was also the case for the other forced frequency, $\mu_{0}=6.4$. The reason is not the fact that the frequencies are forced but rather that they are very low. In fact, for all frequencies with $\mu_{0}<11$, the acoustic wavelength exceeds the spanwise width of the simulation, which might be a reason for the significant underprediction of the acoustic pressure using the three-dimensional acoustic analogy. The two-dimensional theory is independent of the spanwise width because it only uses the information from the lateral plane at $y_{3}=0.725$. For frequencies sufficiently high to be accommodated by the integration domain, the amplitudes predicted by the three-dimensional acoustic analogy appear to be more useful, as shown in figure $14(b-d)$. For $\mu_{0}=16.2$ the amplitude from both the two- and three-dimensional acoustic analogy are comparable and are within $5 \mathrm{~dB}$ of the DNS data. Also, the directivity pattern is very similar. For even higher frequencies, the three-dimensional theory yields amplitudes considerably larger than the two-dimensional predictions, which leads to a superior comparison with the DNS data. In addition to a better prediction of amplitudes, the directivity pattern obtained from the three-dimensional theory agrees better with the DNS data for higher frequencies.

Overall, for low frequencies the single-frequency two-dimensional theory produces nearly the same directivity patterns as the computationally more intensive threedimensional theory. For higher frequencies, however, reasonable agreement with DNS data, i.e. the number and angles of lobes, can only be obtained when using the 

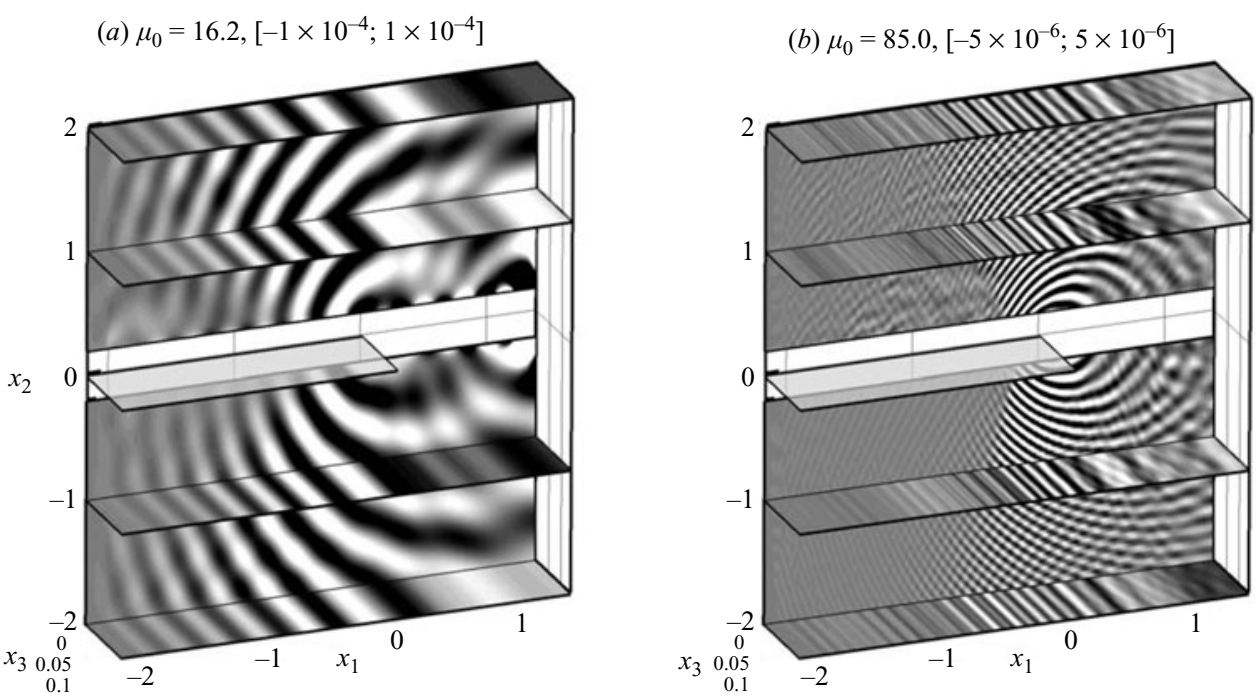

FiguRE 15. Real part of pressure in the frequency domain at planes parallel to the plate, the single plane at $y_{3}=0.725$ is shifted to $x_{3}=0$ for better visibility; the grey shaded surface shows the plate.

three-dimensional acoustic analogy. A possible explanation for this behaviour is explored in the following.

In the two-dimensional single-frequency theory, the spanwise-averaged surface pressure difference, i.e. the zeroth spanwise mode, of a particular frequency is used as input data to calculate the acoustic pressure. One can expect that this is only a good approximation when the surface pressure is highly correlated in the spanwise direction. It was shown in figure 5 that this is not the case when averaging over all frequencies. However, when computing $\mathscr{R}_{p}$ for an individual frequency as

$$
\mathscr{R}_{p}\left(x_{1}, \Delta_{3}, \omega\right)=\left\langle\frac{p\left(x_{1}, x_{3}, \omega\right) \bar{p}\left(x_{1}, x_{3}+\Delta_{3}, \omega\right)}{p^{2}\left(x_{1}, x_{3}, \omega\right)}\right\rangle
$$

the picture changes. For $\mu_{0}=16.2$, the surface pressure distribution is highly correlated in the spanwise direction, with values of $\mathscr{R}_{p}$ larger than 0.7 over the entire surface. Hence, the streamwise distribution of the surface pressure difference for this frequency at individual spanwise locations is close to the spanwise average. In addition, to evaluate the sound radiation in the far field, contours of the real part of pressure for two frequencies are shown in figure 15 for planes parallel to the surface at $x_{2}= \pm 1,2$. For the lower frequency shown, $\mu_{0}=16.2$, the sound field shows no spanwise variation, suggesting predominantly two-dimensional sound radiation. The significant level of spanwise correlation of the surface pressure and the quasi-two-dimensional sound radiation suggest that the acoustic pressure in the far field can be obtained with reasonable accuracy by employing the simplified two-dimensional theory. That this is indeed the case is demonstrated by the similar predictions of the acoustic pressure obtained using either the two- or three-dimensional theory shown in figure 14(b).

For increasing frequencies, the surface pressure field becomes less correlated in the spanwise direction, as illustrated in figure 16. Moreover, a significant spanwise variation of the sound field can be observed, as shown for $\mu_{0}=85.0$ in figure $15(b)$, implying that the strictly two-dimensional theory is not applicable to higher 

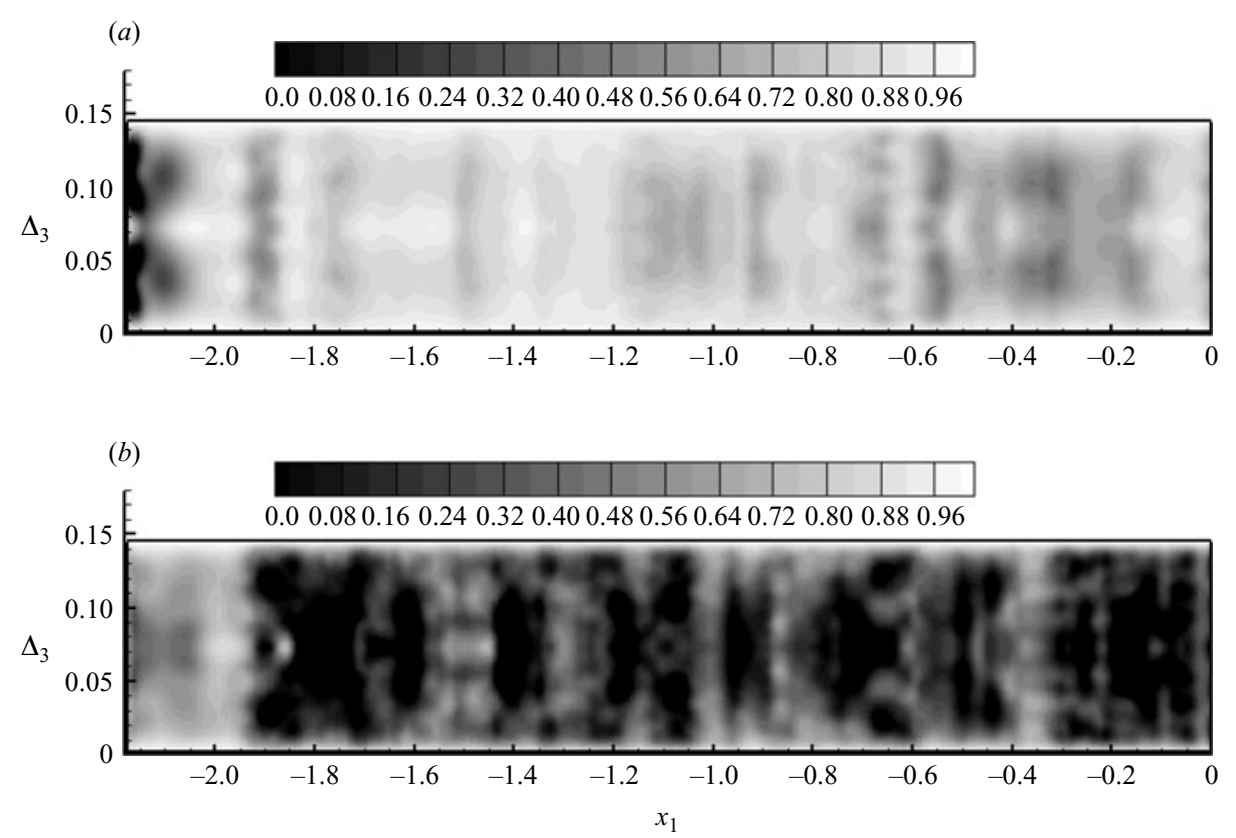

FIGURE 16. Spanwise correlation of pressure on the top surface in the frequency domain. (a) $\mu_{0}=16.2,(b) \mu_{0}=40.5$.

frequencies. This is confirmed by the inability of the two-dimensional acoustic analogy to obtain an acoustic pressure prediction that agrees with the DNS data, shown in figure $14(c, d)$. In conclusion, for low frequencies, the surface pressure is strongly correlated in the spanwise direction andthe sound radiation is predominantly twodimensional, allowing the use of the significantly more cost-effective single-frequency two-dimensional acoustic analogy. For higher frequencies, the spanwise coherence of the surface pressure decreases and the acoustic radiation becomes three-dimensional, demanding the use of the full three-dimensional acoustic analogy.

\subsubsection{Classical theory of Amiet}

In the classical trailing edge noise theory of Amiet (1976b) the far-field sound spectrum is solved according to (2.14). In the derivation of this expression several profound assumptions were made, presented in $\S 2.2 .2$. In this section, the accuracy of each of these assumptions is tested using DNS data.

Amiet's theory assumes that the far-field sound can be evaluated as a function of the total surface pressure difference. Therefore, it is essential that an accurate representation of $\Delta p_{t}$ be available. Amiet's theory relies on the surface pressure jump transfer function $H_{S}$ to determine $\Delta p_{t}$ as a function of the incident pressure $p_{i}$. As described earlier, the current set-up featuring turbulent and laminar boundary layers on the top and bottom surfaces, respectively, allows the computation of the incident pressure field $p_{i}$. Equation (2.10) is then used to compute the total surface pressure difference $\Delta p_{t}$ from $p_{i}$, which can be compared to the total surface pressure difference directly obtained from the DNS data, $\Delta p_{t}=p_{\text {top }}-p_{\text {bot }}$. Hence, the surface pressure difference transfer function of Amiet (1976a), which is one of the key elements of the classical trailing-edge theory, can be evaluated. A comparison between the predicted and the directly computed total surface pressure difference is shown in 

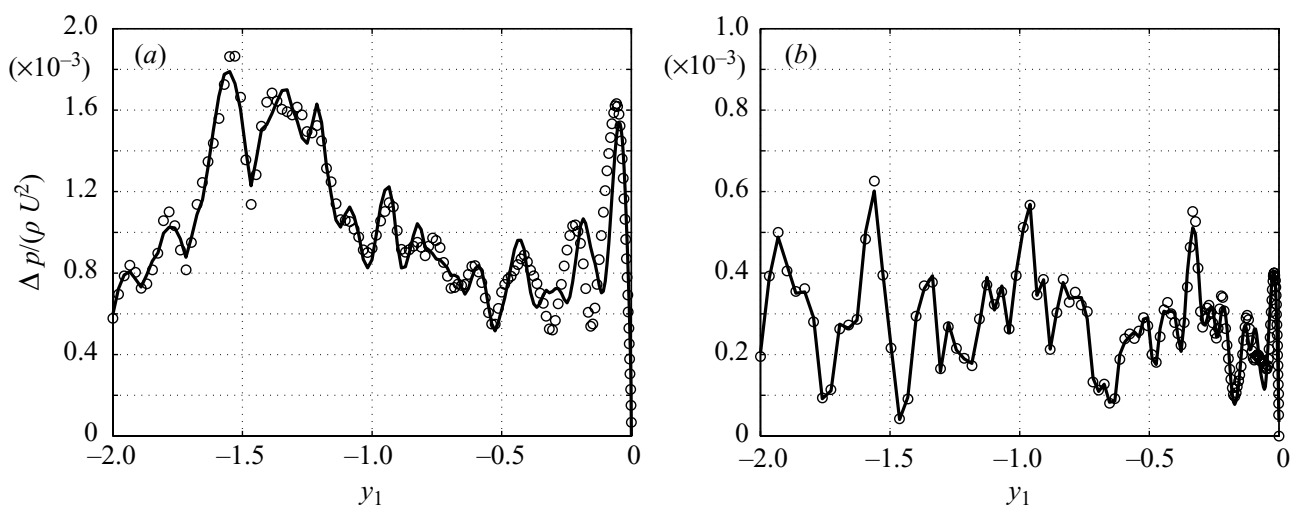

Figure 17. Total surface pressure difference $\Delta p_{t}: \bigcirc$, span average of $\Delta p_{t}=p_{\text {top }}-p_{\text {bot }}$ from DNS; — using Amiet's theory with $H_{D}=\left(1+H_{S}\right)$. (a) $\mu_{0}=16.2,(b) \mu_{0}=40.5$.

figure 17 over the length of the plate for two frequencies. It appears that the agreement between the predicted value of $\Delta p_{t}$ and the DNS data improves for increasing frequency, although the predicted surface pressure difference is satisfactory even at lower frequencies. The good agreement with DNS data implies that obtaining the incident pressure field by subtracting the bottom surface pressure from the top surface pressure is a useful approach. The results are also particularly encouraging because one of the assumptions made when deriving the surface pressure jump function is the validity of the unsteady Kutta condition. As in the two-dimensional trailing-edge simulations conducted by Sandberg et al. (2007), the pressure difference at the trailing edge is non-zero for the turbulent case, although the amplitudes are small. Therefore, the unsteady Kutta condition appears to be a reasonable approximation even for the turbulent flow under consideration.

Another simplification used in the derivation of (2.14) is to treat the turbulence as 'frozen', i.e. the turbulent velocity field is unaffected by the trailing edge and convects past the trailing edge in an unmodified way. As discussed in $\$ 5.1$, the flow changes significantly in the vicinity of the trailing edge. In addition, point spectra of surface pressure difference shown in figure $12(b, c)$ demonstrate that the shape of the spectrum changes over the chord, invalidating the assumption of a frozen spectrum. Amiet's empirical approximation of the surface pressure spectrum (2.16) is shown in figure 18 and compared with the spectra obtained from DNS at the trailing edge and two locations upstream of the TE. Note that (2.16) was multiplied by 2 to convert to a one-sided spectrum. The virtual origin for the calculation of the displacement thickness was specified as $y_{1}=-6.98$ resulting in a trailing-edge displacement thickness equal to the DNS result. The spectra from the simulation change significantly from the upstream positions to the trailing edge, in particular for frequencies lower than $\mu_{0}=100$. However, the shape of the spectra is approximated with reasonable accuracy for the low-frequency range. Overall, the agreement with the DNS data is fair considering that the turbulent boundary layer computed here is at a significantly lower Reynolds number and that the turbulent boundary layer is subject to a pressure gradient close to the trailing edge.

The spanwise correlation length used in (2.14) is approximated as $l_{y_{3}}(\omega)=2.1 U_{c} / \omega$. The DNS data can be used to verify this relation by computing $l_{y_{3}}$ from the definition given in (2.15). Figure 19 shows the spanwise correlation length obtained using (2.15) at three streamwise positions compared with the empirical approximation. 


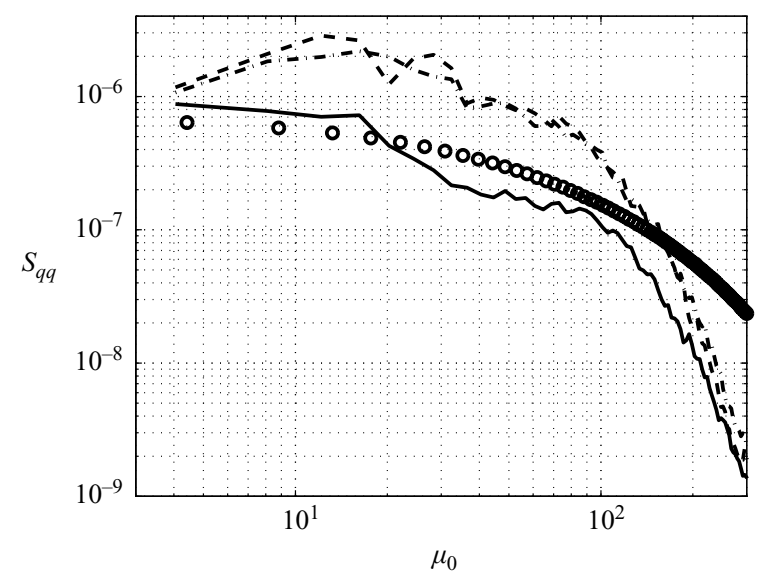

FIGURE 18. Surface pressure spectrum $S_{q q}$ for turbulent boundary layer: $\bigcirc$, empirical approximation of Amiet (1976b), assuming the virtual origin of the turbulent boundary layer at $y_{1}=-6.98 ;-$, DNS at trailing edge; --- , DNS at $y_{1}=-0.36 ;-\cdot-\cdot-$, DNS at $y_{1}=-0.72$.

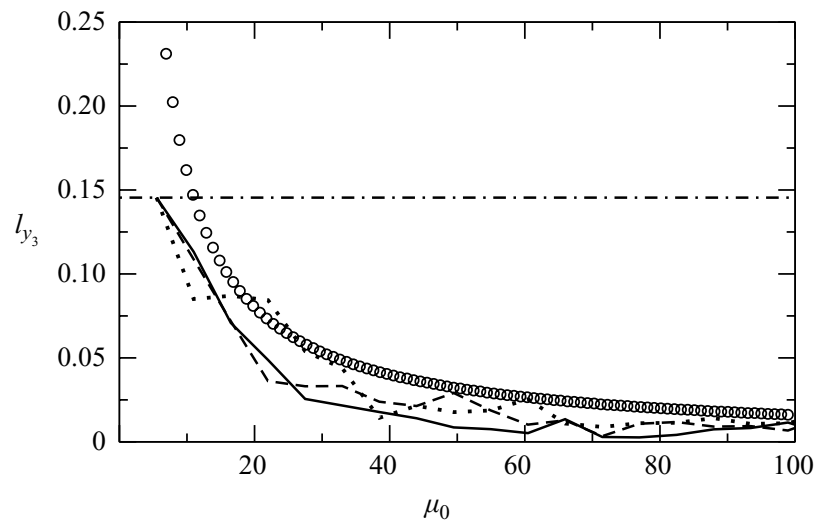

FIGURE 19. Spanwise correlation length $l_{y_{3}}$ for turbulent boundary layer: $O$, empirical approximation of Amiet $(1976 b)$; — DNS at trailing edge; --- , DNS at $y_{1}=-0.36 ; \cdots$, DNS at $y_{1}=-0.72 ;-\cdot-$, spanwise domain width.

For frequencies $\mu_{0}>15$ the approximation suggested by Amiet agrees fairly well with the DNS data and therefore appears to be a reasonable representation of the spanwise correlation length. However, for lower frequencies, the spanwise correlation length obtained from DNS is limited by the spanwise domain width and therefore cannot increase to the large values obtained from the empirical approximation. Therefore, considerable differences in amplitude are expected when comparing the sound pressure level obtained from Amiet's theory with DNS data. It should be mentioned that when computing $l_{y_{3}}$ from the DNS data, the solution varied slightly depending on the number of segments used for the ensemble average. The larger the number of segments used, the better the agreement with Amiet's approximation became, although the low frequencies could not be captured.

A further assumption in the derivation of (2.14) is the far-field approximation $x_{i} \gg y_{i}$. In order to evaluate the near-field effects, the acoustic pressure was evaluated 

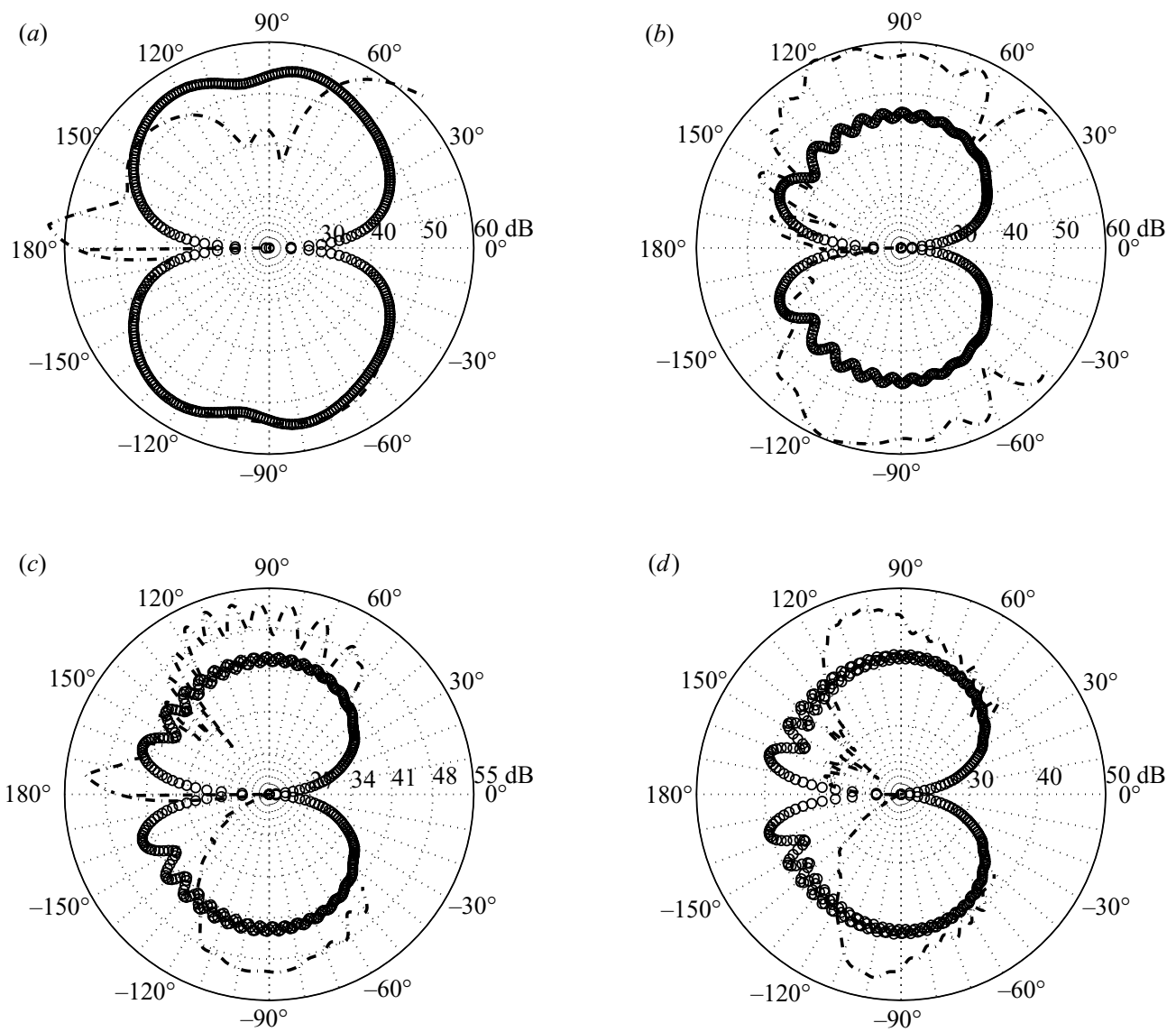

FIGURE 20. Magnitude of acoustic pressure in $\mathrm{dB}$ at the lateral plane with $y_{3}=0.725:-\cdot-$, $|p|$ DNS; $\bigcirc$, full theory of Amiet; $R_{d}=1.75$. (a) $\mu_{0}=3.2$, (b) $\mu_{0}=16.2$, (c) $\mu_{0}=26.3$, (d) $\mu_{0}=40.5$.

at various radii from the trailing edge using the two-dimensional acoustic analogy, which does not invoke the far-field approximation. The directivity shape did not significantly change for $R_{d}>1.75$, implying that near-field effects are reasonably small for the radius chosen.

Having scrutinized the main assumptions made in the derivation of the classical trailing-edge theory, it now remains to compare the prediction of the acoustic pressure using (2.14) with the DNS results, shown in figure 20. Note that (2.14) was multiplied by $4 \pi$ to account for a one-sided spectrum and the conversion to a $1 \mathrm{~Hz}$ bandwidth. For the lowest frequency investigated good agreement with the DNS data is achieved on the lower side, both in terms of directivity and amplitude. For all higher frequencies the amplitude is overpredicted by the DNS. This can be explained as follows. As already mentioned, the periodic boundary conditions employed in the DNS prevent the acoustic waves from leaving the computational domain. Even though this can be interpreted as conducting a simulation with an infinite spanwise extent, a fundamental difference to the infinite span assumption made by Amiet should be pointed out. For the case of a plate with infinite span the turbulence is only correlated over a small spanwise extent. Figure 19 confirms that the spanwise correlation length is smaller than the spanwise extent of the simulation for frequencies greater than $\mu_{0}=12$. All 

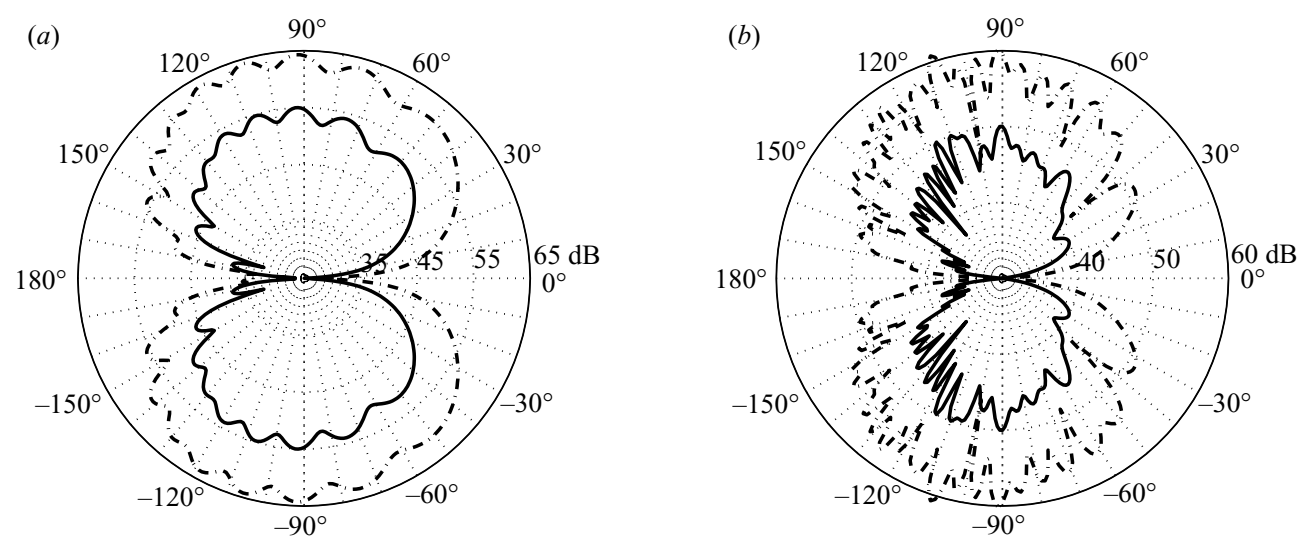

Figure 21. Magnitude of acoustic pressure $|p|$ in $\mathrm{dB}$ at $y_{3}=0.0725$ obtained using the three-dimensional acoustic analogy: - , using sources in the computational domain; $-\cdot-\cdot-$, using sources in the computational domain and two additional mirror images; $R_{d}=1.75$. (a) $\mu_{0}=16.2,(b) \mu_{0}=40.5$.

sources located at a distance greater than the correlation length from the lateral plane at $y_{3}=0.725$ will therefore not be correlated with the turbulence at that plane and hence no significant interference is expected. In contrast, the periodic boundary conditions used for the DNS result in a spanwise array of mirror images which are perfectly correlated. This produces a significant amount of interference that will increase the sound level at $y_{3}=0.0725$. To illustrate this phenomenon, the acoustic pressure was computed with the three-dimensional acoustic analogy, accounting for the sound radiation of the sources of two mirror images, one placed on each side of the computational domain, i.e. (2.6) was integrated from $-3 / 2 d$ to $3 / 2 d$ in the spanwise direction. Figure 21 illustrates the difference between the two cases for two frequencies, showing an increase of up to $10 \mathrm{~dB}$ when using the additional mirror images. The number of mirror images contributing significantly to the sound level at $y_{3}=0.0725$ will be frequency dependent; nevertheless it can be concluded that the interference of the sound waves remaining within the computational domain considerably increases the sound level of the DNS data.

For higher frequencies the directivity shape also deviates from the DNS results in the upstream direction, i.e. $120^{\circ} \leqslant|\theta| \leqslant 180^{\circ}$. The differences are more significant than when using the three-dimensional acoustic analogy, shown in figure 14. This indicates that, although the individual approximations made by Amiet were shown to be reasonable, they collectively produce a noise prediction that significantly deviates from the DNS data, in particular in the upstream direction at higher frequencies.

Nevertheless, overall the classical theory of Amiet is able to predict the acoustic pressure with reasonable accuracy, despite the considerable number of assumptions and simplifications. The differences in directivity and amplitude compared with DNS are most likely not due to the assumptions made in the derivation of the classical theory. On the contrary, comparison with DNS data revealed that Amiet's assumptions, even though not satisfied exactly, can be considered to be good ones. Only at higher frequencies does the interaction of all assumptions result in differences between the predicted acoustic pressure and the DNS data in the upstream direction. More generally, the origin of the differences between the prediction of Amiet's classical theory and the DNS data can therefore most likely be attributed to a more fundamental level of the theory, namely relating the sources on the surface to the 
far-field sound using a Kirchhoff-type integration. This is confirmed by the fact that results obtained with the three-dimensional acoustic analogy do not provide significantly improved results. Some of the observed differences between the predictions and DNS data might be attributed to the fact that individual frequencies were scrutinized. In a theoretical study of airfoil noise, Evers \& Peake (2002) found that the airfoil geometry can have a significant effect on individual frequency components, while the broadband noise is relatively unaffected due to cancellation effects when integrating over the entire spectrum. The authors suspect that this cancellation effect might also occur for the flat plate. Furthermore, it was discussed above that the use of periodic boundary conditions in the simulations, not accounted for in any theory, is responsible for differences in amplitude.

\section{Conclusion}

Direct numerical simulations were conducted of turbulent flow past a trailing edge in order to investigate the hydrodynamic near field and the trailing edge noise generation. A turbulent inflow generation technique was employed on the top side of the flat plate to produce a turbulent boundary layer that convects downstream and reaches $R e_{\delta_{T E}^{*}}=2222$ at the trailing edge. It is demonstrated that the turbulent boundary layer is well resolved by the numerical simulation; however, some discrepancies between the DNS data and an incompressible reference DNS exist. These are mostly due to the inflow sponge zone required to reduce acoustic contamination of the region of interest by the turbulent inflow forcing. In the vicinity of the trailing edge, the skin friction increases significantly and the displacement thickness and pressure fluctuations decrease. It is demonstrated that the behaviour of the flow in the neighbourhood of the trailing edge can be computed with reasonable accuracy with analytical predictions using triple-deck theory when the turbulent eddy viscosity is taken into account. The behaviour of the lower-surface laminar boundary layer at the trailing edge does not compare well with analytic predictions because the pressure field is strongly modified by the turbulent boundary layer on the top side.

The DNS data were Fourier transformed in time and point spectra of surface pressure difference and pressure in the radiation field were scrutinized. The amplitudes of the spectra at the trailing edge are considerably lower than farther upstream, in particular for low frequencies. It is suggested that this is due to the thinning of the boundary layer and the associated increase in the skin friction coefficient at the trailing edge. Composite visualizations of the real part of the spanwise vorticity component and pressure in frequency space illustrate that the wavelength of the vorticity corresponds to the acoustic wavelength. For several frequencies, additional noise sources on the top side lead to an asymmetric sound field. In those cases, the acoustic analogies and Amiet's theory fail to reproduce the DNS data on both sides of the trailing edge because they are based on the surface pressure difference and therefore inherently predict a symmetric radiation pattern.

It is demonstrated that for low frequencies, the single-frequency two-dimensional acoustic analogy produces reasonable agreement with both DNS and the computationally more expensive three-dimensional theory. This can be attributed to the fact that the surface pressure is strongly correlated in the spanwise direction and the sound radiation is predominantly two-dimensional. Once the level of spanwise coherence decreases, the sound radiation becomes three-dimensional and acoustic pressure predictions by strictly two-dimensional variants of theory deteriorate, necessitating the use of the full three-dimensional theory. 
The accuracy of the assumptions made by Amiet for the derivation of the classical trailing-edge theory were tested using DNS data. Amiet's surface pressure jump transfer function is found to provide a good prediction of the total surface pressure difference when using the spanwise average of the surface pressure data. It was shown that pressure spectra change significantly in the streamwise direction, contradicting Amiet's assumption of frozen turbulence. However, Amiet's empirical approximation of the surface pressure spectrum approaches the shape of the spectra from DNS with adequate accuracy for the low-frequency range. Amiet's expression for the spanwise correlation length was found to be a good approximation when compared with DNS data of turbulent flow in the vicinity of a trailing edge. Overall, despite several profound assumptions, the classical trailing-edge theory of Amiet predicts the acoustic pressure with reasonable accuracy. Only at higher frequencies do Amiet's assumptions collectively lead to a mismatch between the predicted acoustic pressure and the DNS data in the upstream direction. Differences in amplitude of 5-10dB between the results obtained with the classical theory and the DNS data are mainly attributed to the periodic boundary conditions in the spanwise direction used for the simulations. The discrepancies in directivity, in particular for higher frequencies, suggest that future work should investigate approaches based on integration surfaces placed outside the boundary layer that might potentially yield more accurate noise predictions.

This work was supported by the DTI under the MSTTAR DARP programme. Computer time was provided by the EPSRC grant GR/S27474/01. The authors would also like to thank Dr Phil F. Joseph from the ISVR for many useful discussions and suggestions concerning the classical trailing-edge theory.

\section{REFERENCES}

Amiet, R. 1975 Acoustic radiation from an airfoil in a turbulent stream. J. Sound Vib. 41 (4), 407-420.

Amiet, R. 1976a High-frequency thin airfoil theory for subsonic flow. AIAA J. 14 (8), 1076-1082.

Amiet, R. $1976 b$ Noise due to turbulent flow past a trailing edge. J. Sound Vib. 47 (3), 387-393.

AmiEt, R. 1978 Effect of the incident surface pressure field on noise due to turbulent flow past a trailing edge. J. Sound Vib. 57 (2), 305-306.

Bodony, D. \& Lele, S. 2002 Spatial scale decomposition of shear layer turbulence and the sound sources associated with the missing scales in a large-eddy simulation. AIAA Paper 2002-2454.

Brooks, T. \& Hodgson, T. 1981 Trailing edge noise prediction from measured surface pressures. J. Sound Vib. 78 (1), 69-117.

Carpenter, M. H., Nordström, J. \& Gottlieb, D. 1999 A stable and conservative interface treatment of arbitrary spatial accuracy. J. Comput. Phys. 148 (2), 341-365.

Colonius, T., Lele, S. K. \& Moin, P. 1993 Boundary conditions for direct computation of aerodynamic sound generation. AIAA J. 31 (9), 1574-1582.

Daniels, P. 1977 Viscous mixing at a trailing edge. J. Mech. Appl. Maths 30 (3), 319-342.

Evers, I. \& Peake, N. 2002 On sound generation by the interaction between turbulence and a cascade of airfoils with non-uniform mean flow. J. Fluid Mech. 463, 25-52.

Ffowcs Williams, J. \& Hall, L. 1970 Aerodynamic sound generation by turbulent flow in the vicinity of a scattering half plane. J. Fluid Mech. 40 (4), 657-670.

GaJJAR, J. \& TürKYILMAZOG̃LU, M. 2000 On the absolute instability of the triple-deck flow over humps and near wedged trailing edges. Phil. Trans. R. Soc. Lond. A 358, 3113-3128.

Goldstein, M. E. 1976 Aeroacoustics, 1st edn. McGraw-Hill.

Howe, M. S. 1978 A review of the theory of trailing edge noise. J. Sound Vib. 61 (3), 437-465.

Hu, Z. W., Morfey, C. L. \& Sandham, N. D. 2006 Wall pressure and shear stress spectra from direct simulations of channel flow. AIAA J. 44 (7), 1541-1549. 
Lighthill, M. $1952 a$ On sound generated aerodynamically I. General theory. Proc. R. Soc. Lond. A 211, 564-587.

Lighthill, M. $1952 b$ On sound generated aerodynamically II. Turbulence as a source of sound. Proc. R. Soc. Lond. A 222, 1-32.

Manoha, E., Herrero, C., Sagaut, P. \& Redonnet, S. 2002 Numerical prediction of airfoil aerodynamic noise. AIAA Paper 2002-2573.

Marsden, O., Bogey, C. \& Bailly, C. $2006 a$ Direct noise computation around a 3-D NACA0012 airfoil. AIAA Paper 2006-2503.

Marsden, O., Bogey, C. \& Bailly, C. $2006 b$ Direct noise computation around a 3-D NACA0012 airfoil. AIAA Paper 2005-2817.

Messiter, A. F. 1970 Boundary-layer flow near the trailing edge of a flat plate. SIAM J. Appl. Maths 18 (1), 241-257.

Oberai, A., Roknaldin, F. \& Hughes, J. 2002 Computation of trailing-edge noise due to turbulent flow over an airfoil. AIAA J. 40 (11), 2206-2216.

SAndberg, R. D., Jones, L. E. \& SAndham, N. D. 2006 A zonal characteristic boundary condition for numerical simulations of aerodynamic sound. In European Conference on Computational Fluid Dynamics, ECCOMAS CFD 2006 (ed. P. Wesseling, E. Oñate \& J. Périaux).

SANDberG, R. D. \& SANDham, N. D. 2006 Nonreflecting zonal characteristic boundary condition for direct numerical simulation of aerodynamic sound. AIAA J. 44 (2), 402-405.

SANDBerg, R. D., SANDham, N. D. \& JosePh, P. F. 2007 Direct numerical simulations of trailing-edge noise generated by boundary-layer instabilities. J. Sound Vib. 304 (3-5), 677-690.

Sandham, N., LI, Q. \& YeE, H. 2002 Entropy splitting for high-order numerical simulation of compressible turbulence. J. Comput. Phys. 178, 307-322.

Sandham, N., Yao, Y. \& LaWal, A. 2003 Large-eddy simulation of transonic flow over a bump. Int. J. Heat Fluid Flow 24, 584-595.

Schlichting, H. 1979 Boundary Layer Theory, 7th edn. McGraw-Hill.

Seror, C., Sagaut, P., Bailly, C. \& Juvé, D. 2001 On the radiated noise computed by large-eddy simulation. Phys. Fluids 13, 476-487.

Singer, B., Brentner, K., Lockard, D. \& Lilley, G. 2000 Simulation of acoustic scattering from a trailing edge. J. Sound Vib. 230 (3), 541-560.

Smith, F. T. 1982 On the high reynolds number theory of laminar flows. IMA J. Appl. Maths 28 (3), 207.

SpalaRT, P. R. 1988 Direct simulation of a turbulent boundary layer up to $r_{\theta}=1410$. J. Fluid Mech. 187, 61-98.

Spalding, D. B. 1961 A single formula for the law of the wall. Trans. ASME: J. Appl. Mech. 28 (3), 444-458.

Stewartson, K. 1968 On the flow near the trailing edge of a flat plate. Proc. R. Soc. Lond. A 306, $275-290$.

WANG, M. \& MoIN, P. 2000 Computation of trailing-edge flow and noise using large-eddy simulation. AIAA J. 38 (12), 2201-2209.

White, F. M. 1991 Viscous Fluid Flow. McGraw Hill. 